RESEIVED

ORNL/TM-13519
OAK RIDGE NATIONAL LABORATORY
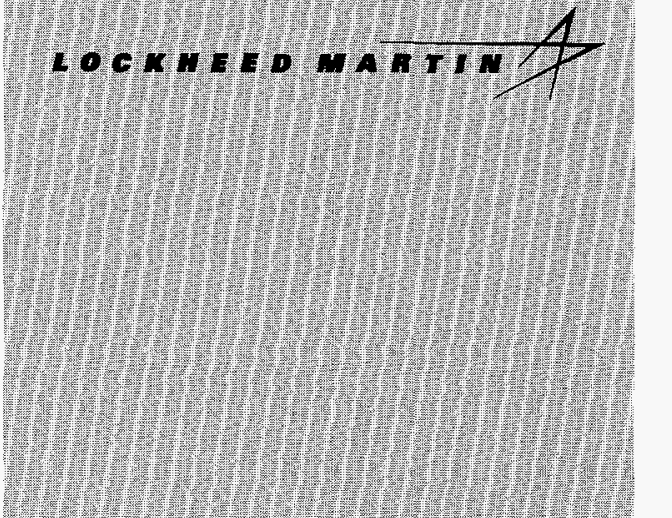

(1)
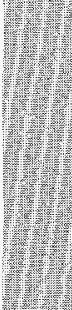

A)

अain

\section{(4)}

MANAGED AND OPERATEO BY

LOCKHEED MARTIN ENERGY BESEARCH CORPORATION FOA THE UNTEO STATES

DEPAMUUENT OF ENERGY

\section{Tensile and Compressive Behavior of a Swirl Mat Composite}

\author{
M. B. Ruggles
}


ORNL/TM-13519

\title{
TENSILE AND COMPRESSIVE BEHAVIOR OF A SWIRL MAT COMPOSITE
}

\author{
M. B. Ruggles
}

July 1998

\author{
Prepared by the \\ OAK RIDGE NATIONAL LABORATORY \\ Oak Ridge, Tennessee 37831-6285 \\ managed by \\ LOCKHEED MARTIN ENERGY RESEARCH CORPORATION \\ for the \\ U.S. DEPARTMENT OF ENERGY \\ under contract DE-AC05-96OR224564
}





\section{DISCLAIMER}

This report was prepared as an account of work sponsored by an agency of the United States Government. Neither the United States Government nor any agency thereof, nor any of their employees, makes any warranty, express or implied, or assumes any legal liability or responsibility for the accuracy, completeness, or usefulness of any information, apparatus, product, or process disclosed, or represents that its use would not infringe privately owned rights. Reference herein to any specific commercial product, process, or service by trade name, trademark, manufacturer, or otherwise does not necessarily constitute or imply its endorsement, recommendation, or favoring by the United States Government or any agency thereof. The views and opinions of authors expressed herein do not necessarily state or reflect those of the United States Government or any agency thereof. 


\section{DISCLAIMER}

Portions of this document may be illegible in electronic image products. Images are produced from the best available original document. 


\section{CONTENTS}

LIST OF FIGURES

LIST OF TABLES . V

ABSTRACT

1. INTRODUCTION

MATERIAL AND SPECIMENS

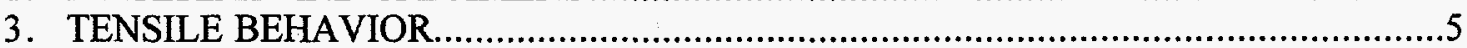

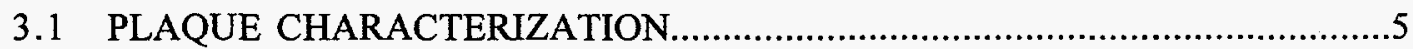

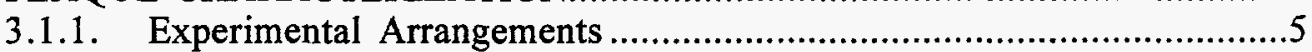

3.1.1.1 Experimental arrangements 1 (EA1) ................................

3.1.1.2 Experimental arrangements 2 (EA2) .............................

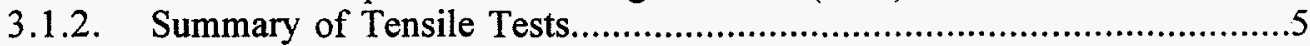

3.2 BEHAVIORAL TRENDS - CORRELATIONS BETWEEN TENSILE

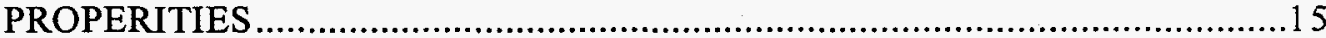

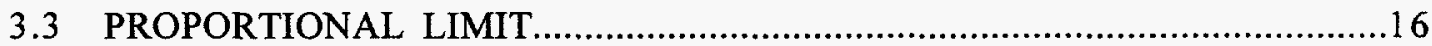

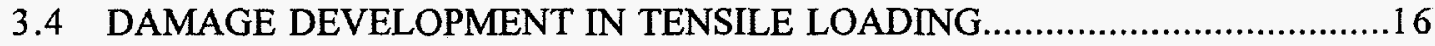

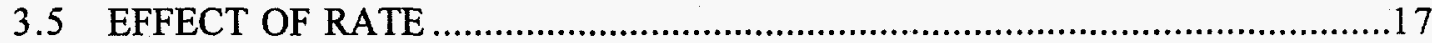

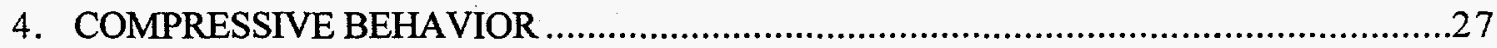

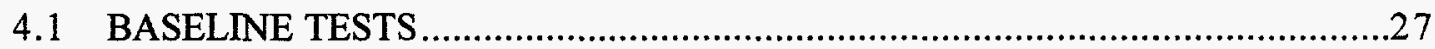

4.1.1. Experimental Arrangements .....................................................22

4.1.2 Summary of Compressive Tests .................................................27

4.2 BEHAVIORAL TRENDS CORRELATIONS BETWEEN COMPRESSIVE

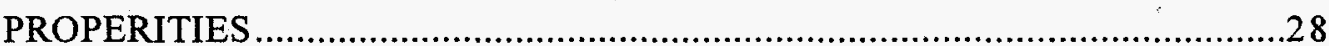

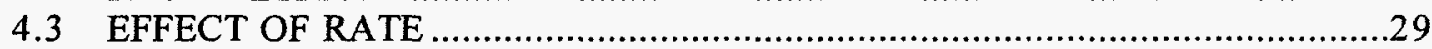

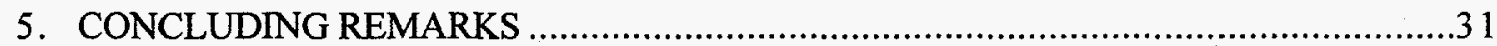

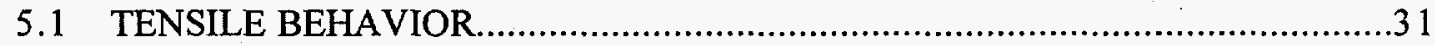

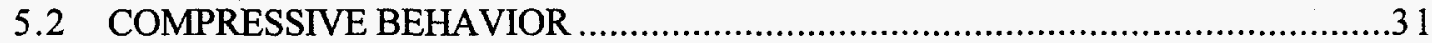

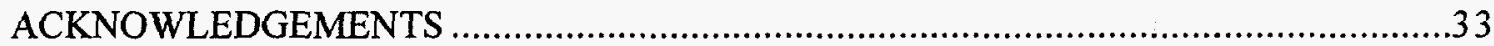

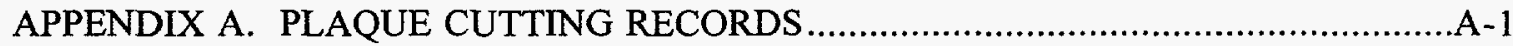





\section{LIST OF FIGURES}

Figure

Page

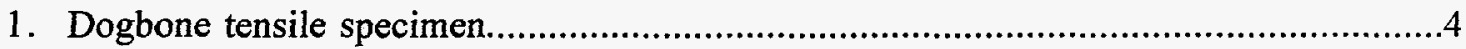

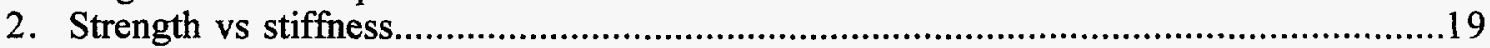

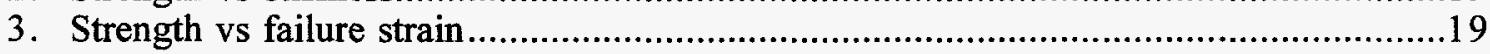

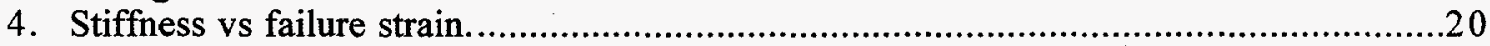

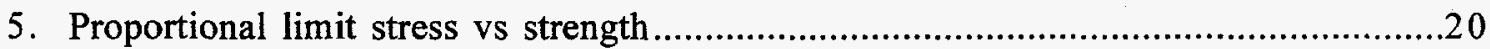

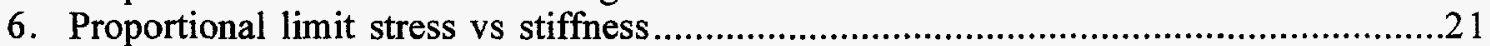

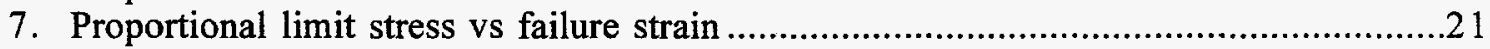

8. Proportional limit stress normalized by stiffness vs strength.................................22

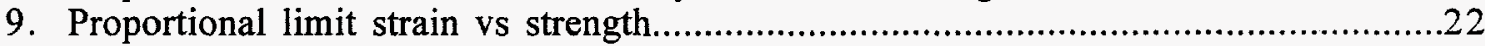

10. Percent change in stiffness vs prior maximum load given in terms of $\%$ UTS ..............23

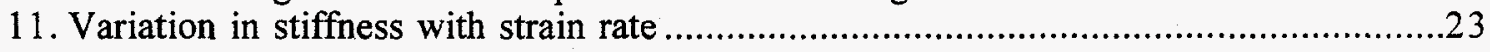

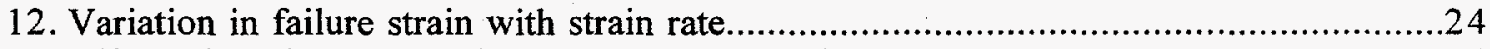

13. Effect of strain rate on ultimate tensile strength..............................................24

14. Stress-strain curves obtained at the strain rates of $10^{-6}, 10^{-4}, 10^{-2}$, and $10 \mathrm{~s}^{-1} \ldots \ldots \ldots \ldots \ldots . .25$

15. Normalized stress-strain curves at the strain rates of $10^{-6}, 10^{-4}, 10^{-2}$, and $10 \mathrm{~s}^{-1} \ldots \ldots \ldots \ldots . . .26$

16. Variation in ultimate tensile strength normalized by stiffness with strain rate............26

17. Compressive stress-strain curves obtained at the load rates of 13980,2330 , and 7.5 $\mathrm{lb} / \mathrm{min}$ corresponding to the strain rates of $2 \times 10^{-3}, 3 \times 10^{-4}$, and $10^{-6} \mathrm{~s}^{-1}$, respectively $\ldots . .30$ 


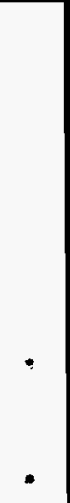




\section{LIST OF TABLES}

Table

1. Summary of tensile tests in the $0^{\circ}$-direction conducted at room temperature in air environment..

2. Summary of tensile tests in the $90^{\circ}$-direction conducted at room temperature in air environment..

3. Summary of plaque average tensile properties in the $0^{\circ}$-direction

4. Summary of plaque average tensile properties in the $90^{\circ}$-direction...............................15

5. Summary of tensile properties obtained at different constant strain rates....................17

6. Summary of compressive tests conducted at room temperature in an air environment 27

7. Summary of compressive properties obtained at different constant load rates 


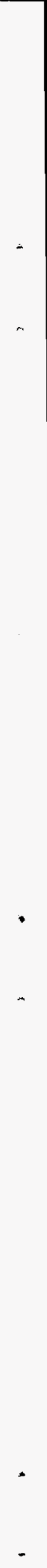




\section{CONVERSION FACTORS}

\section{U.S. Customary Units to SI Units}

\begin{tabular}{llll}
\hline \multicolumn{1}{c}{ Quantity } & \multicolumn{1}{c}{ Multiply } & \multicolumn{1}{c}{ By } & \multicolumn{1}{c}{ To obtain } \\
\hline Length & Inch (in.) & $2.54 \times 10^{-2}$ & Meter $(\mathrm{m})$ \\
Force & Pound $(\mathrm{lb})$ & 4.448 & Newton $(\mathrm{N})$ \\
Stress & Pound $/$ inch $^{2}(\mathrm{psi})^{*}$ & $6.895 \times 10^{6}$ & Pascal $(\mathrm{Pa})$ \\
Temperature & Temperature $\left({ }^{\circ} \mathrm{F}-32\right)$ & 0.5556 & Temperature $\left({ }^{\circ} \mathrm{C}\right)$ \\
\hline
\end{tabular}

${ }^{*} \mathrm{ksi}=10^{3} \mathrm{psi} ; \mathrm{Msi}=10^{6} \mathrm{psi}$ 


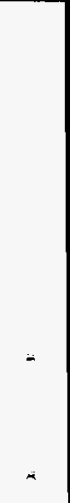




\title{
TENSILE AND COMPRESSIVE BEHAVIOR OF A SWIRL MAT COMPOSITE
}

\author{
M. B. Ruggles
}

\begin{abstract}
The Durability of Lightweight Composite Structures Project was established at Oak Ridge National Laboratory (ORNL) by the U.S. Department of Energy to provide the experimentally-based, durability-driven design guidelines necessary to assure long-term structural integrity of automotive composite components. The initial focus of the ORNL Durability Project was on one representative reference material -an isocyanurate (polyurethane) reinforced with continuous strand, swirl-mat E-glass. The present report describes tensile and compressive testing and results for the reference composite. Behavioral trends and proportional limit are established for both tension and compression. Damage development due to tensile loading and strain rate effects are discussed.
\end{abstract}

\section{INTRODUCTION}

Development of lighter weight, more fuel efficient automobiles represents a technology area where advanced materials can be successfully applied. The fuel efficiency of automobiles can be significantly enhanced by using lightweight materials, such as polymer matrix composites, in primary structural components. However, while significant effort is being devoted to material development and processing, commercial application of composite materials lags behind due, in part, to the lack of specific design guidance and an understanding of the material performance under actual service conditions. There is a recognized need for improved structural design methods and criteria that address deformation and failure behavior of composite materials.

The Durability of Lightweight Composite Structures Project was established at Oak Ridge National Laboratory (ORNL) by the U.S. Department of Energy to provide the experimentally-based, durability-driven design guidelines necessary to assure long-term structural integrity of automotive composite components. The ORNL Durability Project initially focused on characterizing and understanding the mechanical behavior of one candidate composite material system-an SRIM (structural reaction injection molding) isocyanurate (polyurethane) reinforced with continuous strand, swirl-mat E-glass. The present report describes tensile and compressive testing and results produced on the reference isocyanurate composite.

Material and specimen designs are presented in Chapter 2. Chapter 3 is dedicated to tensile behavior. Experimental arrangements are described and tensile test results are summarized and assessed with the purpose of establishing behavioral trends and proportional limit. In addition, damage development due to tensile loading and strain rate effects are discussed. Chapter 4 focuses on compressive behavior, giving the details of experimental arrangements, a summary of tests and test results, as well as offering discussion of behavioral trends and proportional limit in compression. Concluding remarks are made in Chapter 5. 


\section{MATERIAL AND SPECIMENS}

The reference material used in this study is an isocyanurate (polyurethane) reinforced with a continuous-strand, E-glass mat. The resin is DOW MM 364, and the reinforcing is Certainteed/Vetrotex Unifilo U750. The material was provided by the Automotive Composites Consortium (ACC) in 1/8-in.thick plaques, formed by structural reaction injection molding (SRIM). Specimens were cut from plaques both in the rolled direction of the mat, referred to as the $0^{\circ}$ direction, and at a $90^{\circ}$ angle to the rolled direction, referred to as the $90^{\circ}$ direction. The location of the numbered specimens in each of the plaques is shown in appendix A. Flat specimens with tabs were used in the tension and compression tests. BF Goodrich A-1177-B1-B2 two part adhesive was employed to attach the tabs to each end of the specimens for gripping. Specimen and tab dimensions were as follows:

\section{Tension}

Specimen: $\quad 1.0$ in. $\times 0.125$ in. $\times 8.0$ in.

Tabs: $\quad 1.0$ in. $\times 0.125$ in. $\times 2.0$ in.

\section{Compression}

1.0 in. $\times 0.125$ in. $\times 5.25$ in.

1.0 in. $\times 0.0625$ in. $\times 2.25$ in.

In addition to straight-sided tabbed specimens, untabbed dogbone-shaped specimens were used in a small number of tensile tests. The dogbone specimen is shown in Fig. 1. 


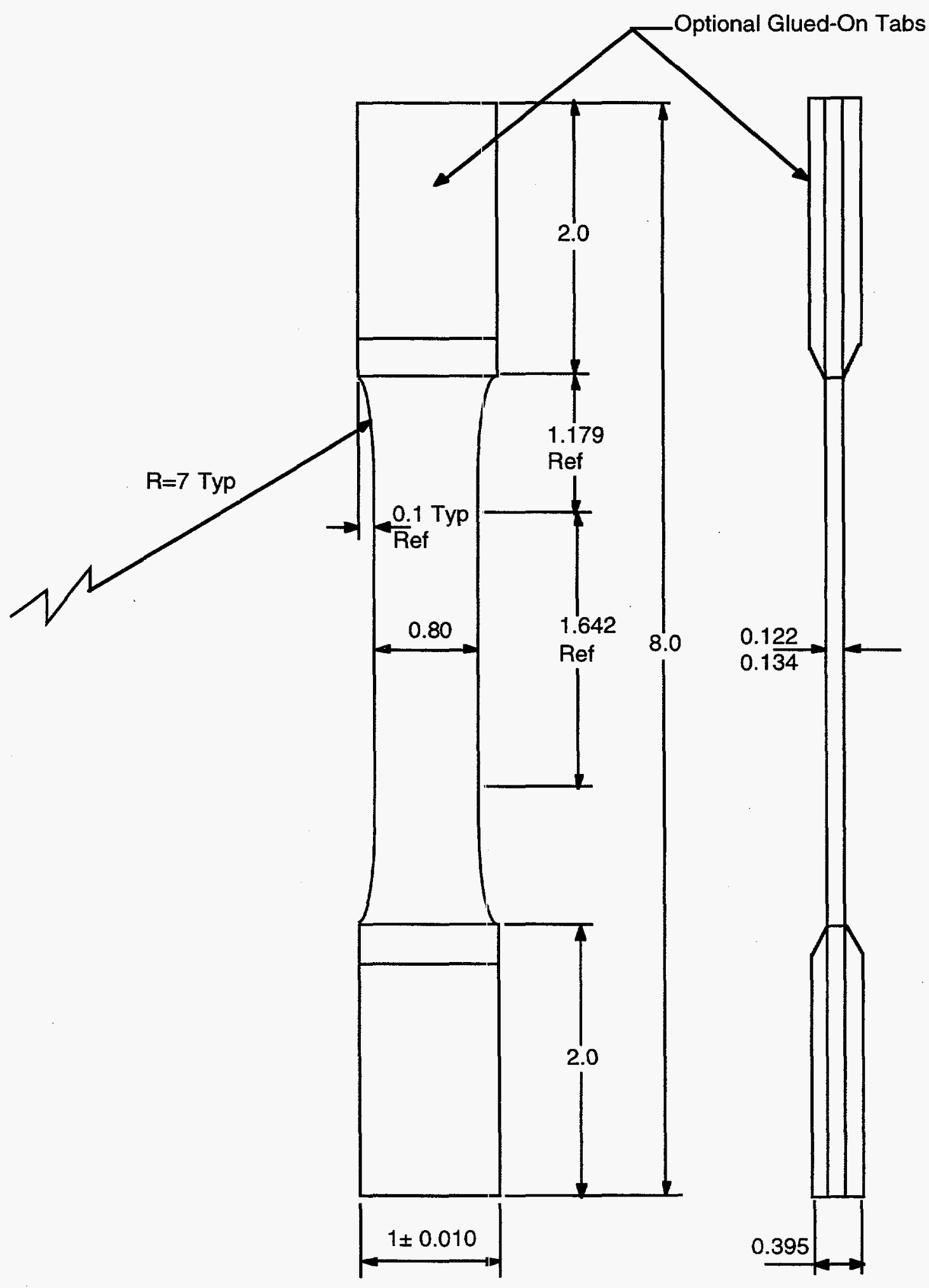

Fig. 1. Dogbone tensile specimen. 


\section{TENSILE BEHAVIOR}

\subsection{PLAQUE CHARACTERIZATION}

\subsubsection{Experimental Arrangements}

All tests were performed at room temperature in an air environment. Two different testing machines, control systems, and testing procedures were employed in tensile tests presented in this report. Both sets of experimental arrangements are summarized below.

\subsubsection{Experimental arrangements 1 (EA1)}

A servocontrolled MTS axial-torsion mechanical testing machine together with an MTS digital TestStar Materials Testing Workstation was used for computerized testing and data acquisition. The load (engineering stress), strain, and displacement were measured and recorded; the digitized test data were stored on both hard and floppy disks. The data acquisition intervals were established on the basis of load, i.e., a data point was recorded whenever the load changed by $10 \mathrm{lb}$. After the test the digitized data can be recalled for processing and interpretation. The entire history is available for analysis.

Specimens were mounted in Instron mechanical wedge grips. Strain measurement was accomplished with an MTS 632.11B-20 extensometer of 1.0 in. gage length. To accurately measure elastic modulus $\mathrm{E}$, tensile tests were preceded with three load controlled cycles between $100 \mathrm{lb}$ and $500 \mathrm{lb}$ (elastic regime) conducted at a load rate of $40 \mathrm{lb} / \mathrm{s}(240 \mathrm{lb} / \mathrm{min})$. Then the elastic modulus was established as the average $\mathrm{E}$ obtained during cycling. Tensile tests to failure were conducted in displacement control at a rate of $0.001 \mathrm{in} . / \mathrm{s}$.

\subsubsection{Experimental arrangements 2 (EA2)}

A servocontrolled MTS axial mechanical testing machine together with an MTS 407 controller and an MTS 418.91 Microprofiler function generator was used for automated testing. The data acquisition was accomplished with a Hewlett Packard 3852, a data acquisition and control unit. Both control and data acquisition systems were interfaced with a Macintosh Power PC with a LabView development system. Strain in the gage section was measured with an MTS 632-11F-20 extensometer with a 1.0 in. gage length. Specimens were mounted in Curtis 5 kip mechanical wedge grips and loaded to failure in displacement control at a rate of $0.001 \mathrm{in} / \mathrm{s}$. Load (engineering stress), strain, and displacement were monitored and recorded when any transducer signal changed by $5 \mathrm{mv}$. Elastic modulus was calculated using linear regression for the initial stress-strain slope from 0 to 4000 psi.

Results presented below demonstrate that both sets of experimental arrangements generated consistent data. In addition, it should be noted that while EAl offer a more certain measurement of the elastic modulus, both sets of experimental arrangements produced consistent stiffness measurements.

\subsubsection{Summary of Tensile Tests}

Tensile test results and test conditions are summarized in Tables 1 and 2 , for the $0^{\circ}$ direction and $90^{\circ}$ direction specimens, respectively. The summary of test conditions includes specimen type , i.e. straight-sided (S) or dogbone (D), and test method, namely experimental arrangements 1 (EA1) or 2 (EA2) defined in Section 3.1.1. The summary of test results includes the elastic modulus, E, ultimate tensile strength, UTS, failure strain, $\varepsilon_{f}$, failure location, and proportional limit stress and strain. Also given in Tables 1 and 2 are the overall average, $A$, standard deviation, $S$, and coefficient of variation, $C_{v}$, for each of the tensile properties. The coefficient of variation is defined as $C_{V}=(S / A) \times 100 \%$ and gives some measure of the scatter of a particular data set. In the failure location column, 1 denotes a tab failure, 2 denotes failure within 1 in. of a tab, and 3 denotes failure more than 1 in. away from the tab or the point of reduction in cross-sectional area of a dogbone specimen. It should be noted that failure was assumed to occur at the point of the ultimate tensile strength. Thus, the failure strain is the strain corresponding to the ultimate tensile strength. Proportional limit was defined as the point where the tensile stress-strain curve departed from linearity. The following procedure was adopted to calculate proportional limit stress and strain values. (i) Establish the elastic stiffness value based on the stresses and strains in the elastic region $(0.8 \mathrm{ksi}<$ stress $<4 \mathrm{ksi})$. 
(ii) Calculate "running" stiffness for each pair of the stress-strain values forming the remaining stressstrain curve.

(iii) Divide the "running" stiffness by the elastic stiffness. The stress-strain point where the stiffness ratio becomes, and remains, less than 1.0 is the proportional limit.

Specimen numbers contain reference to the plaque number and $0^{\circ}$ or $90^{\circ}$ direction. Tensile specimens with numbers containing letters $L$ or $R$ were cut from impact plate specimens according to the cutting plan shown in Fig. A.8 of appendix A. Thus, number P1:-1 $4 \mathrm{~L}$ refers to the tensile specimen $4 \mathrm{~L}$ cut from impact specimen 1 from plaque 11.

Table 1. Summary of tensile tests in the $0^{\circ}$-direction conducted at room temperature in air environment

\begin{tabular}{|c|c|c|c|c|c|c|c|c|}
\hline $\begin{array}{l}\text { Specimen } \\
\text { Number }\end{array}$ & $\begin{array}{l}\text { Specimen } \\
\text { Type }\end{array}$ & $\begin{array}{c}\text { Test } \\
\text { Method }\end{array}$ & $\begin{array}{c}\text { Stiffness } \\
\text { (Msi) }\end{array}$ & $\begin{array}{c}\text { Ultimate } \\
\text { Tensile } \\
\text { Strength } \\
\text { (ksi) }\end{array}$ & $\begin{array}{l}\text { Failure } \\
\text { Strain (\%) }\end{array}$ & $\begin{array}{l}\text { Proportional } \\
\text { Limit Stress } \\
\text { (ksi) }\end{array}$ & $\begin{array}{l}\text { Proportional } \\
\text { Limit Strain } \\
(\%)\end{array}$ & $\begin{array}{l}\text { Failure } \\
\text { Location }\end{array}$ \\
\hline $\begin{array}{l}\text { P1-0-5 } \\
\text { P1-0-11 }\end{array}$ & $\begin{array}{l}S \\
S\end{array}$ & $\begin{array}{l}\text { EAl } \\
\text { EA1 }\end{array}$ & $\begin{array}{l}1.28 \\
1.39\end{array}$ & $\begin{array}{l}21.0 \\
24.0\end{array}$ & $\begin{array}{l}2.37 \\
2.43\end{array}$ & $\begin{array}{c}4.78 \\
-\end{array}$ & $\begin{array}{c}0.38 \\
-\end{array}$ & $\begin{array}{l}2 \\
3\end{array}$ \\
\hline P1-0-20 & $\mathrm{S}$ & EA1 & 1.34 & 19.6 & 2.13 & 5.08 & 0.38 & 2 \\
\hline P2-0-5 & $\mathrm{S}$ & EA1 & 1.22 & 16.0 & 1.75 & 3.18 & 0.26 & 1 \\
\hline P2-0-11 & $\mathrm{S}$ & EA1 & 1.38 & 21.4 & 2.19 & 4.85 & 0.36 & 3 \\
\hline P2-0-20 & $S$ & EA1 & 1.21 & 18.3 & 2.15 & 3.92 & 0.33 & 3 \\
\hline P3-0-5 & $\mathrm{S}$ & EAl & 1.52 & 19.8 & 1.82 & 3.56 & 0.24 & 1 \\
\hline P3-0-11 & S & EA1 & 1.47 & 23.9 & 2.19 & 6.51 & 0.46 & 3 \\
\hline P3-0-20 & $S$ & EA1 & 1.41 & 21.9 & 2.12 & 4.11 & 0.30 & 1 \\
\hline P4-0-5 & S & EAl & 1.44 & 21.0 & 2.03 & 4.67 & 0.33 & 1 \\
\hline P4-0-11 & $S$ & EA1 & 1.33 & 23.6 & 2.36 & 4.58 & 0.35 & 2 \\
\hline P4-0-20 & $S$ & EA1 & 1.38 & 21.6 & 2.14 & 6.02 & 0.44 & 2 \\
\hline P5-0-5 & $\mathrm{s}$ & EAl & 1.31 & 18.6 & 1.94 & 4.53 & 0.35 & 1 \\
\hline P5-0-11 & $\mathrm{S}$ & EA1 & 1.25 & 16.8 & 1.72 & 3.53 & 0.29 & 2 \\
\hline P5-0-20 & S & EA1 & 1.22 & 17.8 & 2.12 & 4.15 & 0.35 & 3 \\
\hline P6-0-1 & $S$ & EAl & 1.35 & 20.8 & 1.99 & 5.23 & 0.39 & 1 \\
\hline P6-0-29 & $S$ & EAl & 1.28 & 19.1 & 2.10 & 4.46 & 0.35 & 1 \\
\hline P6-0-30 & $S$ & EAl & 1.39 & 18.9 & 1.92 & 4.60 & 0.34 & 1 \\
\hline P6-0-58 & $S$ & EA1 & 1.40 & 19.2 & 1.93 & 5.00 & 0.37 & 1 \\
\hline P8-0-23 & $\mathrm{S}$ & EAl & 1.32 & 24.0 & 2.08 & 8.81 & 0.67 & 1 \\
\hline P8-0-32 & S & EA1 & 1.55 & 21.0 & 1.76 & 5.62 & 0.39 & 2 \\
\hline P9-0-31A & $\mathrm{S}$ & EA1 & 1.58 & 20.1 & 0.89 & 5.60 & 0.21 & 1 \\
\hline P9-0-30A & $S$ & EA1 & 1.60 & 19.7 & 1.07 & 4.74 & 0.19 & 1 \\
\hline P11-0-1 & S & EA1 & 1.57 & 21.6 & 1.81 & 4.64 & 0.30 & 1 \\
\hline P11-0-3 & $\mathrm{S}$ & EA1 & 1.31 & 20.0 & 2.26 & - & - & 3 \\
\hline P11-0-8 & S & EA1 & 1.23 & 19.5 & 2.25 & 4.82 & 0.39 & 1 \\
\hline P12-0-1 & $S$ & EA1 & 1.21 & 19.2 & 2.23 & 5.00 & 0.42 & 1 \\
\hline P12-0-3 & $S$ & EA1 & 1.34 & 19.5 & 2.13 & 5.54 & 0.42 & 3 \\
\hline P12-0-8 & $S$ & EAl & 1.28 & 21.0 & 2.42 & 4.45 & 0.35 & 1 \\
\hline P13-0-1 & $\mathrm{S}$ & EA1 & 1.35 & 18.5 & 1.98 & 5.52 & 0.42 & 1 \\
\hline P13-0-3 & $S$ & EA1 & 1.28 & 19.0 & 2.06 & 3.54 & 0.28 & 3 \\
\hline P13-0-8 & S & EA1 & 1.45 & 20.6 & 1.98 & 4.79 & 0.34 & 1 \\
\hline P14-0-1 & S & EAl & 1.40 & 21.2 & 2.09 & 3.99 & 0.29 & 1 \\
\hline P14-0-3 & $\mathrm{s}$ & EA1 & 1.51 & 19.8 & 1.85 & 5.64 & 0.38 & 3 \\
\hline P14-0-4 & $\mathrm{S}$ & EA1 & 1.21 & 19.3 & 2.36 & 5.11 & 0.43 & 1 \\
\hline P15-0-1 & $S$ & EAl & 1.28 & 19.1 & 2.25 & 4.33 & 0.35 & 1 \\
\hline
\end{tabular}


Table 1. Continued

\begin{tabular}{|c|c|c|c|c|c|c|c|c|}
\hline $\begin{array}{l}\text { Specimen } \\
\text { Number }\end{array}$ & $\begin{array}{c}\text { Specimen } \\
\text { Type }\end{array}$ & $\begin{array}{c}\text { Test } \\
\text { Method }\end{array}$ & $\begin{array}{l}\text { Stiffness } \\
\text { (Msi) }\end{array}$ & $\begin{array}{c}\text { Ultimate } \\
\text { Tensile } \\
\text { Strength } \\
\text { (ksi) }\end{array}$ & $\begin{array}{c}\text { Failure } \\
\text { Strain (\%) }\end{array}$ & $\begin{array}{l}\text { Proportional } \\
\text { Limit Stress } \\
\text { (ksi) }\end{array}$ & $\begin{array}{c}\text { Proportional } \\
\text { Limit Strain } \\
\text { (\%) }\end{array}$ & $\begin{array}{c}\text { Failure } \\
\text { Location }\end{array}$ \\
\hline P15-0-3 & $\bar{S}$ & EA1 & 1.31 & 19.4 & 2.17 & 4.45 & 0.34 & 1 \\
\hline P15-0-8 & S & EA1 & 1.28 & 20.7 & 2.37 & 7.43 & 0.58 & 3 \\
\hline P21-0-1 & S & EA1 & 1.45 & 21.3 & 1.86 & 5.02 & 0.35 & 1 \\
\hline P21-0-49 & $S$ & EA1 & 1.42 & 24.4 & 2.42 & 5.37 & 0.38 & 3 \\
\hline$P 21-0-50$ & S & EA1 & 1.43 & 22.0 & 2.13 & 4.62 & 0.33 & 1 \\
\hline $\mathrm{P} 21-0-51$ & $S$ & EA1 & 1.46 & 24.2 & 2.38 & 5.43 & 0.38 & 2 \\
\hline$P 21-0-52$ & S & EA1 & 1.42 & 24.2 & 2.47 & 5.46 & 0.39 & 3 \\
\hline $\mathrm{P} 21-0-53$ & $S$ & EA1 & 1.43 & 22.3 & 2.19 & 5.12 & 0.37 & 1 \\
\hline P21-0-54 & $S$ & EA1 & 1.47 & 24.8 & 2.29 & 6.36 & 0.45 & 1 \\
\hline P21-0-55 & $S$ & EA1 & 1.38 & 25.4 & 2.50 & 4.63 & 0.34 & 1 \\
\hline P21-0-56 & $S$ & EA1 & 1.38 & 22.3 & 2.06 & 5.39 & 0.40 & 3 \\
\hline P21-0-29 & $S$ & EA1 & 1.34 & 22.9 & 2.46 & 4.63 & 0.35 & 3 \\
\hline P20-0-1 & $S$ & EA1 & 1.38 & 21.3 & 2.01 & 5.78 & 0.42 & 1 \\
\hline P20-0-28 & $S$ & EA1 & 1.63 & 22.5 & 1.93 & 5.46 & 0.33 & 2 \\
\hline P20-0-29 & $S$ & EA1 & 1.48 & 24.7 & 2.41 & 4.87 & 0.34 & 3 \\
\hline P20-0-30 & $S$ & EA1 & 1.49 & 23.6 & 2.25 & 4.36 & 0.29 & 1 \\
\hline P20-0-56 & $S$ & EA1 & 1.44 & 21.8 & 2.07 & 5.43 & 0.38 & 3 \\
\hline P11-1 4L & $S$ & EA2 & 1.59 & 21.9 & 2.00 & 5.26 & 0.34 & 1 \\
\hline P11-1 3L & $S$ & EA2 & 1.47 & 22.3 & 2.07 & 4.51 & 0.31 & 1 \\
\hline P11-1 3R & $S$ & EA2 & 1.38 & 20.6 & 1.96 & 3.83 & 0.28 & 1 \\
\hline P11-1 4R & $S$ & $\mathrm{EA} 2$ & 1.33 & 21.7 & 2.09 & 4.80 & 0.36 & 2 \\
\hline P15-1 4L & $S$ & EA2 & 1.22 & 19.1 & 2.17 & 4.90 & 0.40 & 3 \\
\hline P15-1 3L & S & EA2 & 1.28 & 20.6 & 2.11 & 4.67 & 0.37 & 2 \\
\hline P15-1 3R & $S$ & EA2 & 1.27 & 16.8 & 1.80 & 4.70 & 0.37 & I \\
\hline P15-1 4R & $S$ & EA2 & 1.21 & 20.2 & 2.28 & 5.04 & 0.42 & 1 \\
\hline P11-2 4L & $\mathbf{S}$ & $\mathrm{EA} 2$ & 1.28 & 18.3 & 2.04 & 5.28 & 0.43 & 3 \\
\hline P11-2 3L & $S$ & $\mathrm{EA} 2$ & 1.33 & 16.6 & 1.76 & 4.81 & 0.37 & 1 \\
\hline P11-2 3R & $S$ & EA2 & 1.20 & 19.7 & 2.37 & 3.87 & 0.32 & 3 \\
\hline P11-2 4R & $S$ & $\mathrm{EA} 2$ & 1.25 & 19.6 & 2.21 & 4.50 & 0.36 & 1 \\
\hline P15-2 4L & $S$ & EA2 & 1.27 & 21.1 & 2.38 & 4.83 & 0.38 & 1 \\
\hline P15-2 3L & $\mathrm{S}$ & EA2 & 1.28 & 12.9 & 1.22 & 5.39 & 0.42 & 1 \\
\hline P15-2 3R & $\mathrm{S}$ & EA2 & 1.29 & 19.7 & 2.15 & 5.54 & 0.43 & 1 \\
\hline P15-2 4R & $S$ & $\mathrm{EA} 2$ & 1.27 & 20.1 & 2.16 & 5.45 & 0.43 & 1 \\
\hline P11-4 4L & $\mathbf{S}$ & $\mathrm{EA} 2$ & 1.23 & 19.8 & 2.32 & 5.80 & 0.48 & 2 \\
\hline P11-4 3L & $S$ & EA2 & 1.20 & 17.1 & 1.97 & 5.53 & 0.48 & 1 \\
\hline P11-4 3R & $S$ & EA2 & 1.16 & 18.0 & 2.11 & 4.58 & 0.40 & 3 \\
\hline P11-4 4R & $S$ & $\mathrm{EA} 2$ & 1.29 & 19.8 & 2.15 & 5.40 & 0.43 & 2 \\
\hline P11-3 4L & $S$ & $\mathrm{EA} 2$ & 1.39 & 22.6 & 2.28 & 4.49 & 0.33 & 1 \\
\hline P11-3 3L & $S$ & EA2 & 1.46 & 20.5 & 1.82 & 5.13 & 0.35 & 1 \\
\hline P11-3 3R & $S$ & EA2 & 1.57 & 19.9 & 1.65 & 5.91 & 0.38 & 1 \\
\hline P11-3 4R & $\mathrm{S}$ & $\mathrm{EA} 2$ & 1.46 & 18.4 & 1.60 & 4.93 & 0.34 & 1 \\
\hline P15-3 4L & $\mathrm{S}$ & EA2 & 1.32 & 21.2 & 2.22 & 5.15 & 0.39 & 3 \\
\hline P15-3 3L & $\mathrm{S}$ & EA2 & 1.22 & 20.2 & 2.31 & 5.58 & 0.46 & 2 \\
\hline P15-3 3R & $\mathrm{S}$ & EA2 & 1.32 & 19.3 & 2.01 & 4.97 & 0.38 & 1 \\
\hline
\end{tabular}


Table 1. Continued

\begin{tabular}{|c|c|c|c|c|c|c|c|c|}
\hline $\begin{array}{c}\text { Specimen } \\
\text { Number }\end{array}$ & $\begin{array}{l}\text { Specimen } \\
\text { Type }\end{array}$ & $\begin{array}{c}\text { Test } \\
\text { Method }\end{array}$ & $\begin{array}{l}\text { Stiffness } \\
\text { (Msi) }\end{array}$ & $\begin{array}{l}\text { Ultimate } \\
\text { Tensile } \\
\text { Strength } \\
\text { (ksi) }\end{array}$ & $\begin{array}{c}\text { Failure } \\
\text { Strain (\%) }\end{array}$ & $\begin{array}{l}\text { Proportional } \\
\text { Limit Stress } \\
\text { (ksi) }\end{array}$ & $\begin{array}{l}\text { Proportional } \\
\text { Limit Strain } \\
\quad(\%)\end{array}$ & $\begin{array}{c}\text { Failure } \\
\text { Location }\end{array}$ \\
\hline $\mathrm{P} 15-3$ 4R & $\mathrm{S}$ & EA2 & 1.23 & 19.8 & 2.22 & 3.98 & 0.32 & 1 \\
\hline P14-1 4L & $S$ & EA2 & 1.33 & 21.7 & 2.31 & 5.19 & 0.39 & 1 \\
\hline P14-1 3L & $S$ & EA2 & 1.31 & 20.8 & 2.15 & 4.86 & 0.37 & 2 \\
\hline P14-1 3R & $\mathrm{S}$ & $\mathrm{EA} 2$ & 1.34 & 19.8 & 2.02 & 5.71 & 0.43 & 3 \\
\hline P14-1 4R & $\mathrm{S}$ & EA2 & 1.43 & 21.0 & 2.03 & 4.88 & 0.34 & 1 \\
\hline P14-2 4L & $\mathbf{S}$ & EA2 & 1.17 & 18.9 & 2.12 & 4.88 & 0.42 & 1 \\
\hline P14-2 3L & S & EA2 & 1.17 & 19.09 & 2.23 & 5.57 & 0.48 & 3 \\
\hline P14-2 3R & $S$ & EA2 & 1.14 & 17.2 & 2.00 & 4.59 & 0.40 & 3 \\
\hline P14-2 4R & $S$ & EA2 & 1.19 & 18.9 & 2.17 & 5.19 & 0.43 & 2 \\
\hline P12-3 4L & S & EA2 & 1.26 & 17.9 & 1.85 & 4.32 & 0.35 & 2 \\
\hline P12-3 3L & $S$ & EA2 & 1.30 & 20.7 & 2.34 & 4.56 & 0.36 & 3 \\
\hline P12-3 3R & $S$ & EA2 & 1.17 & 18.4 & 2.23 & 4.51 & 0.39 & 1 \\
\hline $\mathrm{P} 12-34 \mathrm{R}$ & $S$ & EA2 & 1.42 & 20.8 & 2.14 & 4.45 & 0.32 & 1 \\
\hline P13-1 4L & S & EA2 & 1.24 & 19.2 & 2.18 & 4.48 & 0.36 & 3 \\
\hline P13-1 3L & $S$ & EA2 & 1.21 & 18.5 & 2.03 & 4.62 & 0.38 & 1 \\
\hline P13-1 3R & $S$ & EA2 & 1.24 & 20.1 & 2.29 & 4.80 & 0.39 & 3 \\
\hline P13-1 4R & $S$ & EA2 & 1.24 & 19.0 & 2.09 & 5.02 & 0.41 & 3 \\
\hline P13-2 4L & $\mathrm{S}$ & $\mathrm{EA} 2$ & 1.18 & 19.5 & 2.21 & 5.30 & 0.45 & 3 \\
\hline P13-2 3L & $S$ & $\mathrm{EA} 2$ & 1.31 & 19.3 & 2.00 & 5.22 & 0.41 & 3 \\
\hline P13-2 3R & $S$ & $\mathrm{EA} 2$ & 1.28 & 19.5 & 2.04 & 5.78 & 0.46 & 3 \\
\hline P13-2 4R & $\mathrm{S}$ & EA2 & 1.28 & 18.5 & 1.97 & 5.47 & 0.43 & 1 \\
\hline P12-1 4L & $S$ & $\mathrm{EA} 2$ & 1.16 & 18.8 & 2.25 & 5.04 & 0.44 & 1 \\
\hline P12-1 3L & $S$ & $\mathrm{EA} 2$ & 1.30 & 19.6 & 2.28 & 4.81 & 0.38 & 3 \\
\hline P12-1 3R & $S$ & $\mathrm{EA} 2$ & 1.19 & 18.3 & 2.22 & 5.38 & 0.46 & 2 \\
\hline P12-1 4R & $S$ & $\mathrm{EA} 2$ & 1.11 & 18.5 & 2.56 & 4.66 & 0.43 & 3 \\
\hline P13-3 4L & $S$ & $\mathrm{EA} 2$ & 1.28 & 19.3 & 2.08 & 4.30 & 0.34 & 2 \\
\hline P13-3 3L & $S$ & EA2 & 1.23 & 18.2 & 2.01 & 4.31 & 0.36 & 1 \\
\hline P13-3 3R & $S$ & EA2 & 1.33 & 19.7 & 2.13 & 4.85 & 0.37 & 1 \\
\hline P13-3 4R & $S$ & EA2 & 1.32 & 20.4 & 2.13 & 5.58 & 0.43 & 1 \\
\hline P12-2 4L & $S$ & EA2 & 1.30 & 18.6 & 2.05 & 5.05 & 0.40 & 2 \\
\hline P12-2 3L & $S$ & EA2 & 1.41 & 19.9 & 2.08 & 4.94 & 0.36 & 1 \\
\hline P12-2 3R & $S$ & EA2 & 1.26 & 19.7 & 2.11 & 5.03 & 0.40 & 1 \\
\hline P12-2 4R & S & EA2 & 1.27 & 18.2 & 1.92 & 4.94 & 0.40 & 2 \\
\hline P13-4 4L & $\mathrm{S}$ & EA2 & 1.31 & 20.6 & 2.34 & 5.34 & 0.42 & 3 \\
\hline P13-4 3L & $S$ & $\mathrm{EA} 2$ & 1.38 & 23.0 & 2.41 & 7.91 & 0.59 & 3 \\
\hline P13-4 3R & $S$ & $\mathrm{EA} 2$ & 1.36 & 21.0 & 2.17 & 5.74 & 0.43 & 1 \\
\hline P13-4 4R & $S$ & $\mathrm{EA} 2$ & 1.28 & 20.6 & 2.34 & 5.69 & 0.45 & 3 \\
\hline P12-4 4L & $S$ & EA2 & 1.20 & 21.0 & 2.50 & 5.22 & 0.44 & 2 \\
\hline P12-4 3L & $S$ & $\mathrm{EA} 2$ & 1.32 & 19.9 & 2.17 & 5.14 & 0.40 & 1 \\
\hline P12-4 3R & $S$ & $\mathrm{EA} 2$ & 1.45 & 20.9 & 2.11 & 5.06 & 0.36 & 1 \\
\hline P12-4 4R & $\mathrm{S}$ & EA2 & 1.16 & 20.1 & 2.33 & 4.53 & 0.39 & 3 \\
\hline P14-3 4L & $\mathrm{S}$ & EA2 & 1.16 & 19.7 & 2.27 & 4.93 & 0.43 & 1 \\
\hline P14-3 3L & $\mathrm{S}$ & EA2 & 1.22 & 21.1 & 2.39 & 4.61 & 0.38 & 3 \\
\hline P14-3 3R & $S$ & EA2 & 1.31 & 21.1 & 2.21 & 3.54 & 0.27 & 1 \\
\hline
\end{tabular}


Table 1. Continued

\begin{tabular}{|c|c|c|c|c|c|c|c|c|}
\hline $\begin{array}{l}\text { Specimen } \\
\text { Number }\end{array}$ & $\begin{array}{l}\text { Specimen } \\
\text { Type }\end{array}$ & $\begin{array}{c}\text { Test } \\
\text { Method }\end{array}$ & $\begin{array}{l}\text { Stiffness } \\
\text { (Msi) }\end{array}$ & $\begin{array}{c}\text { Ultimate } \\
\text { Tensile } \\
\text { Strength } \\
\text { (ksi) }\end{array}$ & $\begin{array}{c}\text { Failure } \\
\text { Strain (\%) }\end{array}$ & $\begin{array}{l}\text { Proportional } \\
\text { Limit Stress } \\
\text { (ksi) }\end{array}$ & $\begin{array}{c}\text { Proportional } \\
\text { Limit Strain } \\
(\%)\end{array}$ & $\begin{array}{c}\text { Failure } \\
\text { Location }\end{array}$ \\
\hline $\begin{array}{l}\text { P14-3 4R } \\
\text { P17-0-1 }\end{array}$ & $\begin{array}{l}S \\
S\end{array}$ & $\begin{array}{l}\text { EA2 } \\
\text { EA1 }\end{array}$ & $\begin{array}{l}1.40 \\
1.46\end{array}$ & $\begin{array}{l}21.0 \\
22.6\end{array}$ & $\begin{array}{l}2.04 \\
2.34\end{array}$ & $\begin{array}{l}4.56 \\
4.38\end{array}$ & $\begin{array}{l}0.33 \\
0.32\end{array}$ & $\begin{array}{l}1 \\
3\end{array}$ \\
\hline P17-0-29 & $\mathrm{S}$ & EA1 & 1.38 & 17.5 & 1.83 & 4.95 & 0.37 & 1 \\
\hline$P 17-0-56$ & $\mathrm{~S}$ & EA1 & 1.30 & 17.4 & 1.80 & 5.69 & 0.45 & 1 \\
\hline P18-0-1 & $S$ & EA1 & 1.44 & 21.4 & 2.02 & 5.42 & 0.39 & 1 \\
\hline P18-0-29 & $\mathrm{s}$ & EA1 & 1.31 & 19.1 & 2.18 & 5.08 & 0.40 & 2 \\
\hline$P 18-0-56$ & $\mathrm{~S}$ & EA1 & 1.27 & 17.5 & 1.93 & 4.99 & 0.40 & 1 \\
\hline P19-0-1 & S & EA1 & 1.30 & 23.1 & 2.45 & 5.13 & 0.40 & 1 \\
\hline P19-0-29 & $\mathrm{S}$ & EA1 & 1.50 & 24.7 & 2.35 & 5.67 & 0.39 & 3 \\
\hline P19-0-56 & $S$ & EA1 & 1.32 & 20.3 & 2.13 & 5.06 & 0.40 & 1 \\
\hline P22-0-1 & $S$ & EA1 & 1.63 & 24.8 & 2.15 & 5.26 & 0.33 & 1 \\
\hline P22-0-3 & $S$ & EA1 & 1.59 & 23.7 & 2.10 & 5.46 & 0.36 & 1 \\
\hline P22-0-5 & $S$ & EA1 & 1.58 & 27.7 & 2.44 & 4.81 & 0.31 & 3 \\
\hline P22-0-7 & $S$ & EA1 & 1.57 & 23.5 & 2.08 & 4.50 & 0.29 & 2 \\
\hline P23-0-1 & $S$ & EA1 & 1.34 & 19.4 & 1.79 & 5.23 & 0.38 & 1 \\
\hline P23-0-3 & S & EA1 & 1.53 & 21.5 & 1.93 & 4.92 & 0.32 & 1 \\
\hline P23-0-6 & $S$ & EA1 & 1.49 & 23.4 & 2.13 & 5.63 & 0.38 & 1 \\
\hline P23-0-8 & $S$ & EA1 & 1.44 & 21.1 & 1.99 & 5.28 & 0.38 & 1 \\
\hline P26-0-1 & $\mathrm{S}$ & EA1 & 1.45 & 21.4 & 1.85 & - & - & 1 \\
\hline P26-0-2 & $S$ & EAI & 1.46 & 21.4 & 2.02 & - & - & 1 \\
\hline P26-0-7 & S & EA1 & 1.49 & 20.6 & 1.90 & - & - & 1 \\
\hline P26-0-8 & $S$ & EAl & 1.37 & 22.2 & 2.16 & - & - & 1 \\
\hline$P 27-0-22$ & $S$ & EAl & 1.52 & 27.9 & 2.00 & - & - & 2 \\
\hline P30-0-1 & $S$ & EAl & 1.40 & 24.0 & 2.29 & - & - & 3 \\
\hline P30-0-2 & $S$ & EA1 & 1.43 & 25.8 & 2.55 & - & - & 2 \\
\hline P30-0-4 & $S$ & EAl & 1.41 & 23.4 & 2.34 & - & - & 1 \\
\hline P30-0-5 & $S$ & EA1 & 1.41 & 24.9 & 2.45 & - & - & 3 \\
\hline P30-0-6 & $\mathrm{S}$ & EAl & 1.43 & 25.1 & 2.36 & - & - & 3 \\
\hline P30-0-7 & $S$ & EA1 & 1.53 & 23.9 & 1.99 & - & - & 3 \\
\hline P30-0-8 & $S$ & EA1 & 1.48 & 24.3 & 2.29 & - & - & 3 \\
\hline P33-0-1 & $\mathrm{S}$ & EA1 & 1.41 & 22.0 & 2.01 & 5.24 & 0.38 & 1 \\
\hline P33-0-28 & S & EA1 & 1.48 & 17.6 & 1.49 & 5.44 & 0.38 & 3 \\
\hline P33-0-29 & $S$ & EAl & 1.45 & 22.6 & 2.08 & 6.02 & 0.42 & 3 \\
\hline P34-0-1 & $S$ & EAl & 1.44 & 21.9 & 1.94 & 5.66 & 0.40 & 1 \\
\hline P34-0-28 & $S$ & EAl & 1.50 & 23.5 & 2.02 & 7.77 & 0.54 & 1 \\
\hline P34-0-29 & $S$ & EAl & 1.50 & 21.2 & 1.80 & 5.12 & 0.35 & 1 \\
\hline P34-0-30 & $S$ & EA1 & 1.57 & 23.8 & 2.03 & 5.98 & 0.40 & 1 \\
\hline P35-0-1 & $S$ & EA1 & 1.49 & 23.5 & 2.02 & 4.71 & 0.32 & 1 \\
\hline P35-0-28 & $\mathrm{S}$ & EA1 & 1.58 & 23.7 & 1.90 & 6.19 & 0.39 & 1 \\
\hline P35-0-29 & $\mathrm{S}$ & EA1 & 1.59 & 23.8 & 2.07 & 4.03 & 0.26 & 1 \\
\hline P35-0-30 & $S$ & EAl & 1.56 & 24.5 & 2.13 & 5.87 & 0.39 & 1 \\
\hline P36-0-29 & D & EA1 & 1.44 & 26.0 & 2.50 & 5.91 & 0.41 & 2 \\
\hline P36-0-30 & $\mathrm{D}$ & EAl & 1.65 & 26.9 & 2.21 & 5.61 & 0.34 & 3 \\
\hline P36-0-31 & $\mathrm{D}$ & EA1 & 1.53 & 28.7 & 2.66 & 6.57 & 0.44 & 3 \\
\hline
\end{tabular}


Table 1. Continued

\begin{tabular}{|c|c|c|c|c|c|c|c|c|}
\hline $\begin{array}{l}\text { Specimen } \\
\text { Number }\end{array}$ & $\begin{array}{l}\text { Specimen } \\
\text { Type }\end{array}$ & $\begin{array}{c}\text { Test } \\
\text { Method }\end{array}$ & $\begin{array}{l}\text { Stiffness } \\
\text { (Msi) }\end{array}$ & $\begin{array}{l}\text { Ultimate } \\
\text { Tensile } \\
\text { Strength } \\
\text { (ksi) }\end{array}$ & $\begin{array}{c}\text { Failure } \\
\text { Strain (\%) }\end{array}$ & $\begin{array}{l}\text { Proportional } \\
\text { Limit Stress } \\
\text { (ksi) }\end{array}$ & $\begin{array}{c}\text { Proportional } \\
\text { Limit Strain } \\
(\%)\end{array}$ & $\begin{array}{l}\text { Failure } \\
\text { Location }\end{array}$ \\
\hline $\begin{array}{l}\text { P36-0-51 } \\
\text { P36-0-52 }\end{array}$ & $\begin{array}{l}\mathrm{D} \\
\mathrm{D}\end{array}$ & $\begin{array}{l}\text { EA1 } \\
\text { EA1 }\end{array}$ & $\begin{array}{l}1.65 \\
1.39\end{array}$ & $\begin{array}{l}26.1 \\
25.2\end{array}$ & $\begin{array}{l}2.45 \\
2.46\end{array}$ & $\begin{array}{l}4.51 \\
4.76\end{array}$ & $\begin{array}{l}0.28 \\
0.34\end{array}$ & $\begin{array}{l}3 \\
3\end{array}$ \\
\hline P36-0-56 & D & EAl & 1.24 & 23.2 & 2.46 & 4.92 & 0.39 & 3 \\
\hline P37-0-1 & $\mathrm{S}$ & EA1 & 1.42 & 23.6 & 2.18 & 5.50 & 0.39 & 1 \\
\hline P37-0-1 & $S$ & EA1 & 1.42 & 23.6 & 2.18 & 5.50 & 0.39 & 1 \\
\hline P37-0-3 & $S$ & EA1 & 1.80 & 26.1 & 0.02 & 5.22 & 0.29 & 1 \\
\hline P37-0-4 & $S$ & EAl & 1.48 & 24.7 & 2.35 & 5.54 & 0.38 & 1 \\
\hline P37-0-5 & $S$ & EAl & 1.44 & 25.0 & 2.50 & 5.71 & 0.40 & 1 \\
\hline P42-0-1 & $\mathrm{S}$ & EAl & 1.44 & 23.6 & 2.13 & 5.62 & 0.40 & 3 \\
\hline P42-0-28 & $S$ & EAl & 1.58 & 25.7 & 2.29 & 4.54 & 0.29 & 3 \\
\hline P42-0-29 & $\mathrm{S}$ & EA1 & 1.60 & 22.2 & 1.92 & 4.49 & 0.28 & 1 \\
\hline P42-0-30 & $\mathrm{S}$ & EAl & 1.43 & 24.2 & 2.33 & 4.68 & 0.33 & 2 \\
\hline P42-0-56 & $S$ & EA1 & 1.33 & 21.6 & 2.17 & 5.66 & 0.44 & 1 \\
\hline P44-0-1 & $\mathrm{D}$ & EA1 & 1.51 & 27.2 & 2.50 & 5.87 & 0.39 & 3 \\
\hline P44-0-3 & $\mathrm{D}$ & EA1 & 1.48 & 26.4 & 2.58 & 5.29 & 0.36 & 3 \\
\hline P44-0-4 & D & EA1 & 1.54 & 27.8 & 2.59 & 5.18 & 0.34 & 3 \\
\hline P44-0-5 & $\mathrm{D}$ & EA1 & 1.31 & 23.6 & 2.48 & 4.78 & 0.37 & 3 \\
\hline Average & & & 1.37 & 21.3 & 2.12 & 5.08 & 0.38 & \\
\hline Standard & Deviation & & 0.13 & 2.65 & 0.30 & 745 & 0.06 & \\
\hline Coeff. of & Variation & $(\%)$ & 9.32 & 12.5 & 14.2 & 14.7 & 15.8 & \\
\hline
\end{tabular}


Table 2. Summary of tensile tests in the $90^{\circ}$-direction conducted at room temperature in air environment

\begin{tabular}{|c|c|c|c|c|c|c|c|c|}
\hline $\begin{array}{c}\text { Specimen } \\
\text { Number }\end{array}$ & $\begin{array}{l}\text { Specimen } \\
\text { Type }\end{array}$ & $\begin{array}{c}\text { Test } \\
\text { Method }\end{array}$ & $\begin{array}{l}\text { Stiffness } \\
\text { (Msi) }\end{array}$ & $\begin{array}{c}\text { Ultimate } \\
\text { Tensile } \\
\text { Strength } \\
\text { (ksi) }\end{array}$ & $\begin{array}{l}\text { Failure } \\
\text { Strain (\%) }\end{array}$ & $\begin{array}{l}\text { Proportional } \\
\text { Limit Stress } \\
\text { (ksi) }\end{array}$ & $\begin{array}{l}\text { Proportional } \\
\text { Limit Strain } \\
\text { (\%) }\end{array}$ & $\begin{array}{c}\text { Failure } \\
\text { Location }\end{array}$ \\
\hline $\begin{array}{l}\text { P1-90-7 } \\
\text { P1-90-16 }\end{array}$ & $\begin{array}{l}\mathrm{S} \\
\mathrm{S}\end{array}$ & $\begin{array}{l}\text { EA1 } \\
\text { EAl }\end{array}$ & $\begin{array}{l}1.59 \\
1.64\end{array}$ & $\begin{array}{l}30.7 \\
28.3\end{array}$ & $\begin{array}{l}2.51 \\
2.22\end{array}$ & $\begin{array}{l}5.27 \\
5.20\end{array}$ & $\begin{array}{l}0.34 \\
0.32\end{array}$ & $\begin{array}{l}3 \\
3\end{array}$ \\
\hline P1-90-22 & $S$ & EA1 & 1.53 & 28.6 & 2.39 & - & - & 2 \\
\hline P1-90-25 & $S$ & EAl & 1.53 & 29.3 & 2.39 & - & - & 2 \\
\hline P2-90-22 & $S$ & EA1 & 1.59 & 19.4 & 1.44 & 6.07 & 0.39 & 2 \\
\hline P2-90-25 & S & EAl & 1.49 & 27.4 & 2.44 & 5.54 & 0.39 & 2 \\
\hline P3-90-7 & $S$ & EAl & 1.60 & 28.9 & 2.26 & 6.86 & 0.44 & 2 \\
\hline P3-90-16 & S & EAl & 1.70 & 27.9 & 2.07 & 4.77 & 0.29 & 3 \\
\hline P3-90-22 & $S$ & EA1 & 1.63 & 27.8 & 2.26 & 3.44 & 0.22 & 3 \\
\hline P3-90-25 & $\mathrm{S}$ & EA1 & 1.57 & 25.7 & 1.96 & 6.40 & 0.41 & 2 \\
\hline P4-90-7 & $S$ & EA1 & 1.74 & 28.1 & 2.08 & 4.73 & 0.28 & 2 \\
\hline P4-90-16 & S & EA1 & 1.59 & 27.8 & 2.28 & 7.55 & 0.48 & 3 \\
\hline P4-90-22 & $\mathrm{S}$ & EA1 & 1.61 & 26.3 & 2.11 & 4.73 & 0.31 & 3 \\
\hline P4-90-25 & $\mathrm{S}$ & EA1 & 1.53 & 24.7 & 1.88 & 7.46 & 0.49 & 2 \\
\hline P5-90-7 & $\mathrm{S}$ & EA1 & 1.56 & 18.6 & 1.37 & 5.64 & 0.37 & 2 \\
\hline P5-90-16 & $S$ & EAl & 1.39 & 16.3 & 1.37 & 6.91 & 0.52 & 3 \\
\hline P5-90-22 & $S$ & EA1 & 1.55 & 16.8 & 1.23 & 7.93 & 0.53 & 3 \\
\hline P5-90-25 & $S$ & EA1 & 1.58 & 20.6 & 1.53 & 5.31 & 0.34 & 3 \\
\hline P6-90-1 & $\mathrm{S}$ & EA1 & 1.72 & 24.0 & 1.76 & 5.72 & 0.34 & 1 \\
\hline P6-90-2 & $\mathrm{S}$ & EA1 & 1.77 & 29.6 & 2.07 & 8.04 & 0.46 & 3 \\
\hline P6-90-6 & $S$ & EA1 & 1.56 & 29.0 & 2.36 & 5.06 & 0.31 & 1 \\
\hline P7-90-1 & $\mathrm{S}$ & EA1 & 1.74 & 29.6 & 2.23 & 4.58 & 0.27 & 1 \\
\hline P7-90-2 & $\mathrm{S}$ & EA1 & 1.61 & 30.7 & 2.51 & 3.79 & 0.24 & 3 \\
\hline P7-90-6 & $\mathrm{S}$ & EA1 & 1.89 & 30.1 & 1.98 & 4.65 & 0.25 & 1 \\
\hline P8-90-1 & $\mathrm{S}$ & EA1 & 1.67 & 29.9 & 2.35 & 5.83 & 0.36 & 1 \\
\hline P8-90-2 & $\mathrm{S}$ & EA1 & 1.60 & 27.0 & 2.04 & 5.65 & 0.36 & 1 \\
\hline P8-90-3 & $\mathrm{s}$ & EA1 & 1.88 & 31.5 & 2.13 & - & - & 2 \\
\hline P8-90-6 & $\mathrm{S}$ & EAl & 1.82 & 31.9 & 2.27 & 5.39 & 0.30 & 1 \\
\hline P9-90-1 & S & EA1 & 1.72 & 29.6 & 2.10 & 8.11 & 0.47 & 1 \\
\hline P9-90-2 & $S$ & EA1 & 1.74 & 28.9 & 2.06 & 8.63 & 0.51 & 1 \\
\hline P11-90-1 & $S$ & EA1 & 1.56 & 24.9 & 1.94 & 7.05 & 0.43 & 1 \\
\hline P11-90-6 & $S$ & EAl & 1.38 & 26.5 & 2.66 & 6.97 & 0.51 & 1 \\
\hline P11-90-7 & $\mathrm{S}$ & EA1 & 1.61 & 26.0 & 2.02 & 6.68 & 0.42 & 1 \\
\hline P12-90-1 & $S$ & EA1 & 1.55 & 28.9 & 2.33 & 6.19 & 0.40 & 3 \\
\hline P12-90-2 & $\mathrm{S}$ & EA1 & 1.64 & 31.0 & 2.41 & 7.71 & 0.47 & 2 \\
\hline P12-90-6 & $\mathrm{S}$ & EA1 & 1.76 & 29.0 & 2.08 & 9.02 & 0.52 & 1 \\
\hline P13-90-1 & $\mathrm{S}$ & EA1 & 1.55 & 25.5 & 2.09 & 4.69 & 0.31 & 3 \\
\hline P13-90-2 & $\mathrm{S}$ & EAl & 1.67 & 26.8 & 2.01 & 9.93 & 0.61 & 2 \\
\hline P13-90-6 & $S$ & EAl & 1.62 & 29.3 & 2.37 & 7.05 & 0.44 & 3 \\
\hline P14-90-1 & $\mathrm{S}$ & EA1 & 1.57 & 28.8 & 2.31 & 6.41 & 0.41 & 1 \\
\hline P14-90-2 & $\mathrm{S}$ & EA1 & 1.72 & 29.7 & 2.11 & 7.91 & 0.46 & 1 \\
\hline P14-90-6 & S & EAl & 1.46 & 27.8 & 2.44 & 6.47 & 0.45 & 1 \\
\hline P15-90-1 & $\mathrm{S}$ & EAl & 1.65 & 31.4 & 2.30 & 4.26 & 0.26 & 1 \\
\hline
\end{tabular}


Table 2. Continued

\begin{tabular}{|c|c|c|c|c|c|c|c|c|}
\hline $\begin{array}{l}\text { Specimen } \\
\text { Number }\end{array}$ & $\begin{array}{l}\text { Specimen } \\
\text { Type }\end{array}$ & $\begin{array}{c}\text { Test } \\
\text { Method }\end{array}$ & $\begin{array}{l}\text { Stiffness } \\
\text { (Msi) }\end{array}$ & $\begin{array}{c}\text { Ultimate } \\
\text { Tensile } \\
\text { Strength } \\
\text { (ksi) }\end{array}$ & $\begin{array}{c}\text { Failure } \\
\text { Strain (\%) }\end{array}$ & $\begin{array}{l}\text { Proportional } \\
\text { Limit Stress } \\
\quad \text { (ksi) }\end{array}$ & $\begin{array}{c}\text { Proportional } \\
\text { Limit Strain } \\
\text { (\%) }\end{array}$ & $\begin{array}{l}\text { Failure } \\
\text { Location }\end{array}$ \\
\hline $\begin{array}{l}\text { P15-90-2 } \\
\text { P15-90-6 }\end{array}$ & $\begin{array}{l}S \\
S\end{array}$ & $\begin{array}{l}\text { EA1 } \\
\text { EA1 }\end{array}$ & $\begin{array}{l}1.76 \\
1.66\end{array}$ & $\begin{array}{l}29.6 \\
28.3\end{array}$ & $\begin{array}{l}2.10 \\
2.25\end{array}$ & $\begin{array}{l}6.29 \\
6.97\end{array}$ & $\begin{array}{l}0.36 \\
0.43\end{array}$ & $\begin{array}{l}3 \\
1\end{array}$ \\
\hline P17-90-1 & $\mathrm{S}$ & EA1 & 1.78 & 31.5 & 2.25 & 5.64 & 0.32 & 3 \\
\hline P17-90-2 & $\mathbf{S}$ & EA1 & 1.73 & 29.3 & 2.05 & 6.04 & 0.36 & 1 \\
\hline P17-90-6 & $\mathbf{S}$ & EA1 & 1.74 & 29.4 & 2.08 & 5.79 & 0.34 & 3 \\
\hline P18-90-1 & $S$ & EA1 & 1.61 & 28.7 & 2.16 & 9.15 & 0.57 & 3 \\
\hline P18-90-2 & $\mathrm{S}$ & EA1 & 1.90 & 33.2 & 2.17 & 6.37 & 0.34 & 1 \\
\hline P18-90-6 & $S$ & EA1 & 1.74 & 30.6 & 2.25 & 7.13 & 0.42 & 1 \\
\hline P19-90-2 & S & EA1 & 1.70 & 26.4 & 2.12 & 5.75 & 0.35 & 1 \\
\hline P19-90-3 & $S$ & EA1 & 1.69 & 27.0 & 2.09 & 5.46 & 0.33 & 1 \\
\hline P19-90-4 & S & EA1 & 1.82 & 28.7 & 1.99 & 5.83 & 0.34 & 1 \\
\hline P19-90-5 & $\mathrm{S}$ & EA1 & 1.69 & 30.9 & 2.35 & 6.55 & 0.40 & 3 \\
\hline P19-90-6 & S & EA1 & 1.82 & 29.0 & 2.10 & 7.39 & 0.42 & 1 \\
\hline P21-90-1 & $S$ & EA1 & 1.87 & 31.6 & 2.33 & 5.99 & 0.33 & 1 \\
\hline P21-90-2 & S & EA1 & 1.75 & 30.1 & 2.25 & 5.51 & 0.32 & 1 \\
\hline P21-90-6 & $S$ & EAl & 1.66 & 28.7 & 2.24 & 6.79 & 0.41 & 3 \\
\hline P23-90-1 & $S$ & EA1 & 1.63 & 27.1 & 2.18 & 5.95 & 0.37 & 1 \\
\hline P23-90-4 & $\mathrm{S}$ & EA1 & 1.72 & 27.4 & 2.10 & 4.67 & 0.28 & 1 \\
\hline P23-90-5 & $\mathrm{S}$ & EA1 & 1.62 & 27.5 & 2.18 & 6.63 & 0.41 & 1 \\
\hline P23-90-8 & $S$ & EA1 & 1.53 & 23.8 & 1.89 & 5.08 & 0.33 & 1 \\
\hline P30-90-4 & $S$ & EA1 & 1.52 & 27.3 & 2.32 & - & - & 2 \\
\hline P30-90-7 & $S$ & EA1 & 1.66 & 32.0 & 2.50 & - & - & 2 \\
\hline P30-90-9 & $S$ & EA1 & 1.50 & 29.2 & 2.50 & - & - & 2 \\
\hline P33-90-1 & S & EA1 & 1.85 & 28.5 & 2.02 & 6.69 & 0.38 & 1 \\
\hline P33-90-2 & $S$ & EA1 & 1.77 & 29.3 & 2.24 & 6.21 & 0.37 & 1 \\
\hline P33-90-3 & S & EA1 & 1.64 & 27.8 & 2.17 & 5.42 & 0.34 & 3 \\
\hline P33-90-4 & $S$ & EA1 & 1.87 & 28.0 & 2.08 & 5.15 & 0.29 & 1 \\
\hline P33-90-5 & $S$ & EAl & 1.93 & 32.0 & 2.31 & 6.70 & 0.37 & 1 \\
\hline P33-90-6 & $S$ & EA1 & 1.73 & 28.9 & 2.12 & 5.40 & 0.32 & 1 \\
\hline P34-90-1 & $S$ & EAl & 1.88 & 31.1 & 1.97 & 6.72 & 0.37 & 1 \\
\hline P34-90-2 & $S$ & EAl & 1.75 & 30.6 & 2.13 & 5.64 & 0.32 & 1 \\
\hline P34-90-3 & $S$ & EA1 & 1.80 & 30.4 & 2.12 & 6.59 & 0.38 & 1 \\
\hline P34-90-4 & $S$ & EA1 & 1.84 & 30.7 & 2.10 & 8.62 & 0.48 & 1 \\
\hline P34-90-5 & $S$ & EA1 & 1.66 & 29.6 & 2.27 & 6.75 & 0.41 & 1 \\
\hline P34-90-6 & $\mathrm{S}$ & EAl & 1.88 & 29.8 & 1.99 & 4.51 & 0.25 & 3 \\
\hline P35-90-1 & $\mathbf{S}$ & EA1 & 1.87 & 29.1 & 2.03 & 7.83 & 0.43 & 1 \\
\hline P35-90-3 & $\mathrm{S}$ & EA1 & 1.68 & 28.5 & 2.05 & 5.13 & 0.31 & 1 \\
\hline P35-90-6 & $\mathrm{S}$ & EA1 & 1.77 & 30.2 & 2.35 & 4.26 & 0.25 & 3 \\
\hline P37-90-2 & $\mathrm{S}$ & EAl & 1.83 & 31.7 & 2.33 & 5.19 & 0.29 & 1 \\
\hline P37-90-4 & $\mathrm{S}$ & EA1 & 1.83 & 31.5 & 2.23 & 5.07 & 0.28 & 3 \\
\hline P37-90-5 & $\mathrm{S}$ & EAl & 1.79 & 32.2 & 2.30 & 5.30 & 0.30 & 3 \\
\hline P42-90-1 & $S$ & EA1 & 1.78 & 32.1 & 2.37 & 6.40 & 0.37 & 2 \\
\hline P42-90-2 & $\mathrm{S}$ & EAl & 1.80 & 32.3 & 2.46 & 6.77 & 0.39 & 3 \\
\hline P42-90-6 & $\mathrm{S}$ & EA1 & 1.80 & 28.9 & 2.12 & 6.16 & 0.35 & 1 \\
\hline
\end{tabular}


Table 2. Continued

\begin{tabular}{lccccccc}
\hline $\begin{array}{c}\text { Specimen } \\
\text { Number }\end{array}$ & $\begin{array}{c}\text { Specimen } \\
\text { Type }\end{array}$ & $\begin{array}{c}\text { Test } \\
\text { Method }\end{array}$ & $\begin{array}{c}\text { Stiffness } \\
(\mathrm{Msi})\end{array}$ & $\begin{array}{c}\text { Ultimate } \\
\text { Tensile } \\
\text { Strength } \\
(\mathrm{ksi})\end{array}$ & $\begin{array}{c}\text { Failure } \\
\text { Strain (\%) }\end{array}$ & $\begin{array}{c}\text { Proportional } \\
\text { Limit Stress } \\
(\mathrm{ksi})\end{array}$ & $\begin{array}{c}\text { Proportional Failure } \\
\text { Limit Strain Location } \\
(\%)\end{array}$ \\
\hline Average & & & 1.69 & 28.4 & 2.15 & 6.19 & 0.37 \\
Standard & Deviation & & 0.12 & 3.19 & 0.25 & 1.27 & 0.08 \\
Coeff. of & Variation & $(\%)$ & 7.10 & 11.2 & 11.6 & 20.5 & 21.6 \\
\hline
\end{tabular}

It is noteworthy that no significant influence of the experimental arrangements or the specimen shape on the tensile properties is observed. The results in Tables 1 and 2 also indicate that the failure location appears to have no effect on the tensile properties. The tensile results demonstrate that $90^{\circ}$ is the "strong" direction, specimens cut in the $90^{\circ}$ direction consistently produced higher average values of the stiffness and the ultimate tensile strength. Average stiffness, E, for the $90^{\circ}$ direction is $23 \%$ higher than the average $\mathrm{E}$ obtained for the $0^{\circ}$ direction. The average ultimate tensile strength measured for the $90^{\circ}$ direction is $34 \%$ higher than the average obtained for the $0^{\circ}$ direction. However, it should be noted that the average failure strains obtained for the two directions are approximately the same (the difference is on the order of $1 \%)$.

The coefficients of variation are given in Tables 1 and 2 to provide some measure of the data scatter. It is seen that similar values of the coefficients of variation were obtained for tensile properties in both the $0^{\circ}$ and the $90^{\circ}$ directions. For both directions stiffness values exhibit the least amount of scatter (coefficients of variation of $9.3 \%$ for the $0^{\circ}$ and $7.1 \%$ for the $90^{\circ}$ direction), followed by UTS (coefficients of variation of $12.5 \%$ for the $0^{\circ}$ and $11.2 \%$ for the $90^{\circ}$ direction) and failure strain (coefficients of variation of $14 \%$ for the $0^{\circ}$ and $11.6 \%$ for the $90^{\circ}$ direction). It is noteworthy that these differences are small.

Tensile test results are recapitulated in Tables 3 and 4 below, where the number of specimens tested from each particular plaque and the plaque averages of the tensile properties $E$, UTS, and $e_{f}$, for the $0^{\circ}$ direction and the $90^{\circ}$ direction, respectively, are given. Plaques are referred to using both the ORNL and the ACC nomenclature. Tables 3 and 4 permit an easy assessment of plaque-to-plaque variations in tensile properties, and "strong" and "weak" plaques can be readily identified. Overall averages of the tensile properties are included at the bottom of Tables 3 and 4 for an easy comparison of the individual plaque behavior with that of the rest of the population. The $0^{\circ}$ direction results in Table 3 indicate that P8, P20, 22, P27, P34-P37, P42, and P44 are the "stronger" plaques, characterized by E $\geq 1.45 \mathrm{Msi}$ and UTS $\geq$ 22.5 ksi. Results in Table 4 readily identify plaques P2 and P5 as the "weak" plaques, with the UTS significantly lower than the average. It can also be seen that in the $90^{\circ}$-direction plaques P7-P9, P17-P19, $\mathrm{P} 21$, P33-P35, P37, and P42 produced stiffness values $\mathrm{E} \geq 1.7 \mathrm{Msi}$, considerably above the average. 
Table 3. Summary of plaque average tensile properties in the $0^{\circ}$-direction

\begin{tabular}{|c|c|c|c|c|}
\hline $\begin{array}{l}\text { Plaque Number } \\
\text { ORNL / ACC }\end{array}$ & $\begin{array}{c}\text { Number of Specimens } \\
\text { Tested }\end{array}$ & $\begin{array}{l}\text { Average Stiffness } \\
\text { (Misi) }\end{array}$ & $\begin{array}{c}\text { Average } \\
\text { UltimateTensile } \\
\text { Strength (ksi) }\end{array}$ & $\begin{array}{l}\text { Average Failure Strain } \\
(\%)\end{array}$ \\
\hline P1 / 227-44-30 & 3 & 1.34 & 21.5 & 2.31 \\
\hline P2 / 227-44-33 & 3 & 1.27 & 18.6 & 2.03 \\
\hline P3 / 227-44-35 & 3 & 1.47 & 21.9 & 2.04 \\
\hline $\mathrm{P} 4 / 227-44-36$ & 3 & 1.38 & 22.1 & 2.18 \\
\hline P5 / 227-44-37 & 3 & 1.26 & 17.7 & 1.93 \\
\hline P6 / 227-44-27 & 4 & 1.36 & 19.5 & 1.99 \\
\hline P8 / 227-44-32 & 2 & 1.49 & 22.5 & 1.92 \\
\hline P9 / 227-44-28 & 2 & 1.43 & 21.0 & 1.96 \\
\hline P11 / 227-45-41 & 19 & 1.37 & 20.4 & 2.25 \\
\hline $\mathrm{P} 12 / 227-45-52$ & 19 & 1.28 & 19.9 & 2.42 \\
\hline P13 / 227-45-46 & 19 & 1.36 & 19.4 & 1.98 \\
\hline P14 / 227-45-62 & 15 & 1.37 & 20.1 & 2.36 \\
\hline P15 / 227-45-61 & 15 & 1.29 & 19.7 & 2.26 \\
\hline P17 / 227-45-47 & 3 & 1.38 & 19.2 & 1.99 \\
\hline $\mathrm{P} 18 / 227-45-40$ & 3 & 1.34 & 19.3 & 2.04 \\
\hline $\mathrm{P} 19 / 16-13-56$ & 3 & 1.37 & 22.7 & 2.31 \\
\hline $\mathrm{P} 20 / 16-13-57$ & 5 & 1.48 & 22.8 & 2.13 \\
\hline P21 / 16-13-59 & 10 & 1.42 & 23.4 & 2.28 \\
\hline $\mathrm{P} 22 / 16-13-65$ & 4 & 1.59 & 24.9 & 2.19 \\
\hline $\mathrm{P} 23 / 16-13-66$ & 4 & 1.45 & 21.4 & 1.96 \\
\hline $\mathrm{P} 26 / 16-13-70$ & 4 & 1.44 & 21.4 & 1.98 \\
\hline $\mathrm{P} 27 /$ 16-13-71 & 1 & 1.52 & 27.9 & 2.00 \\
\hline $\mathrm{P} 30 / 16-13-74$ & 7 & 1.44 & 24.5 & 2.32 \\
\hline $\mathrm{P} 33 / 16-13-87$ & 3 & 1.44 & 20.7 & 1.86 \\
\hline P34 / 16-13-88 & 4 & 1.50 & 22.6 & 1.95 \\
\hline P35 / 16-13-92 & 4 & 1.56 & 23.9 & 2.03 \\
\hline P36 / 16-13-93 & 6 & 1.48 & 26.0 & 2.46 \\
\hline $\mathrm{P} 37 / 16-13-37$ & 5 & 1.53 & 25.0 & 1.87 \\
\hline $\mathrm{P} 42 / 16-13-42$ & 5 & 1.48 & 23.4 & 2.17 \\
\hline P44 / 16-13-44 & 4 & 1.4 .6 & 26.3 & 2.54 \\
\hline $\begin{array}{c}\text { Total Number of } \\
\text { Specimens }\end{array}$ & 185 & & & \\
\hline Overall Average & & 1.37 & 21.3 & 2.12 \\
\hline Standard Deviation & & 0.13 & 2.70 & 0.30 \\
\hline $\begin{array}{l}\text { Coefficient of } \\
\text { Variation (\%) }\end{array}$ & & 9.32 & 12.5 & 14.15 \\
\hline
\end{tabular}


Table 4. Summary of plaque average tensile properties in the $90^{\circ}$-direction

\begin{tabular}{llccc}
\hline Plaque Number & Number of Specimens & Stiffness (Msi) & $\begin{array}{c}\text { UltimateTensile } \\
\text { Strength (ksi) }\end{array}$ & Failure Strain (\%) \\
\hline P1 / 227-44-30 & 4 & 1.57 & 29.2 & 2.38 \\
P2 / 227-44-33 & 2 & 1.54 & 23.4 & 1.94 \\
P3 / 227-44-35 & 4 & 1.63 & 27.6 & 2.14 \\
P4 / 227-44-36 & 4 & 1.61 & 26.7 & 2.09 \\
P5 / 227-44-37 & 4 & 1.52 & 18.1 & 1.37 \\
P6 / 227-44-27 & 3 & 1.68 & 27.5 & 2.06 \\
P7/ 227-45-13 & 3 & 1.75 & 30.1 & 2.24 \\
P8 / 227-44-32 & 4 & 1.70 & 29.6 & 2.22 \\
P9 / 227-44-28 & 2 & 1.73 & 29.9 & 2.23 \\
P11 / 227-45-41 & 3 & 1.52 & 25.8 & 2.21 \\
P12 / 227-45-52 & 3 & 1.65 & 29.6 & 2.27 \\
P13 / 227-45-46 & 3 & 1.61 & 27.2 & 2.16 \\
P14 / 227-45-62 & 3 & 1.58 & 28.8 & 2.29 \\
P15 / 227-45-61 & 3 & 1.69 & 29.8 & 2.22 \\
P17 / 227-45-47 & 3 & 1.75 & 30.1 & 2.13 \\
P18 / 227-45-40 & 3 & 1.75 & 30.8 & 2.19 \\
P19 / 16-13-56 & 5 & 1.74 & 28.4 & 2.13 \\
P21 / 16-13-59 & 3 & 1.76 & 30.1 & 2.27 \\
P23 / 16-13-66 & 4 & 1.63 & 26.4 & 2.09 \\
P30 / 16-13-74 & 4 & 1.57 & 29.5 & 2.44 \\
P33 / 16-13-87 & 6 & 1.80 & 29.1 & 2.16 \\
P34 / 16-13-88 & 6 & 1.80 & 30.4 & 2.10 \\
P35 / 16-13-92 & 3 & 1.77 & 29.2 & 2.14 \\
P37 / 16-13-37 & 3 & 1.81 & 31.8 & 2.29 \\
P42 / 16-13-42 & 3 & 1.79 & 31.1 & 2.32 \\
Total Number of & 88 & & & \\
Specimens & & 1.69 & 28.4 & \\
Overall Average & & 0.12 & 3.19 & \\
Standard Deviation & & & 11.2 & \\
Coefficient of & & & & \\
Variation (\%) & & & & \\
\hline & & & & \\
\hline
\end{tabular}

\subsection{BEHAVIORAL TRENDS-CORRELATIONS BETWEEN TENSILE PROPERTIES}

Tensile properties presented in Section 3.1.2 above were assessed with the purpose of establishing possible behavioral trends and/or correlations governing ultimate tensile strength (UTS), stiffness, and failure strain for the given material. Ultimate tensile strength, stiffness, and failure strain measured in 273 tensile tests $\left(185\right.$ on the $0^{\circ}$ specimens and 88 on the $90^{\circ}$ specimens) were cross-plotted in Figs. $2-4$, where both $0^{\circ}$ and $90^{\circ}$ direction results are presented. Also included in Figs. 2-4 are results obtained in compression tests, which will be discussed in Chapter 4 below. Plots in Figs. 2-4 demonstrate that tensile results obtained for the $0^{\circ}$ and $90^{\circ}$ directions form a consistent set of data. The $90^{\circ}$ results generally fall on the high end of the set forming a natural continuation of the $0^{\circ}$ distribution.

The plot of UTS vs stiffness (see Fig. 2) indicates that UTS increases with increasing stiffness. Moreover, such dependence of UTS on stiffness can be reasonably well approximated with a straight line, yielding the following correlation:

$$
\sigma_{\text {UTS }}=-4666+0.02 \mathrm{E},
$$


where UTS is in ksi and E is in Msi.The plot of UTS vs failure strain (see Fig. 3) shows UTS to be independent of failure strain. Likewise, distribution of stiffness with failure strain shown in Fig. 4 reveals no dependence of stiffness on failure strain. This observation is consistent with the linear correlation existing between UTS and stiffness (Eq. 1). Both stiffness and UTS may be considered independent of failure strain. Figures 3 and 4 also demonstrate tha: failure strain remains fairly constant (and thus independent of UTS or stiffness) for both $0^{\circ}$ and $90^{\circ}$ directions.

\subsection{PROPORTIONAL LIMIT}

Results of 253 tensile tests ( 171 on the $0^{\circ}$ specimens and 82 on the $90^{\circ}$ specimens) were assessed with the purpose of establishing a proportional limit for the reference material. The proportional limit was defined as the point $\left(\sigma_{\mathrm{PL}}, \varepsilon_{\mathrm{PL}}\right)$ in the stress-strain space where the tensile stress-strain curve departs from linearity. Proportional limit stress and strain values vrere established for each of the 253 specimens. The following average values were calculated:
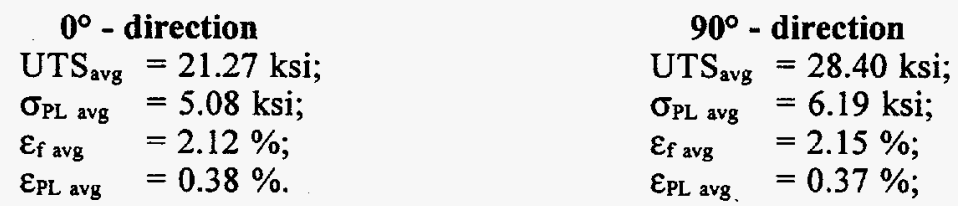

It is seen that the proportional limit stress is $24 \%$ and $22 \%$ of the UTS for the $0^{\circ}$ and $90^{\circ}$ directions, respectively. Proportional limit strain is $18 \%$ and $17 \%$ of the failure strain for the $0^{\circ}$ and $90^{\circ}$ directions, respectively.

To investigate possible trends, proportional limit stress values were plotted vs UTS, stiffness, and failure strain (see Figs. 5-7). In addition, proportional limit stresses were normalized by stiffness and plotted vs strength in Fig. 8. Finally, a plot of proportional limit strain vs strength is given in Fig. 9. Compressive results are included in all plots and will be discussed later in Chapter 4 . Results presented in Fig. 5 indicate that proportional limit stresses increase with increasing UTS. While the increase is not dramatic, it is noticeable and can be approximated with a linear law (a single straight line representing both $0^{\circ}$ and $90^{\circ}$ directions). The following correlation between proportional limit stress and UTS can be proposed:

$$
\sigma_{\mathrm{PL}}=2684+0.012 \sigma_{\mathrm{UTS}} .
$$

It is seen in Fig. 6 that proportional limit stress also increases with stiffness. Again, while not dramatic, the increase is noticeable and can be represented with the following linear correlation:

$$
\sigma_{\mathrm{PL}}=2003+0.002 \mathrm{E} .
$$

Results presented in Fig. 7 demonstrate that, taking specimen to specimen scatter into account, failure strain remains constant for the entire $0^{\circ}-90^{\circ}$ population. Proportional limit stress remains likewise unchanged (with the exception of some data scatter), and thus independent of the failure strain.

Presented in Fig. 8 is the plot of proportional limit stress, normalized by stiffness, vs UTS. Specimen to specimen variations in stiffness have been previously observed and reported. Normalizing proportional limit stress obtained for a particular specimen by stiffness measured for that specimen may offer an opportunity to better assess and compare results produced by specimens with significantly different stiffness values by thus "removing" the influence of stiffness. It is seen in Fig. 8 that proportional limit stress normalized by stiffness remains fairly independent of UTS.

Shown in Fig. 9 is the behavior of the proportional limit strain with changing UTS. It is seen in Fig. 9 that proportional limit strain remains constant (considering data scatter typical for this material) with respect to UTS for the entire $0^{\circ}-90^{\circ}$ population of tensile results. It can be concluded that proportional limit strain is independent of UTS.

\subsection{DAMAGE DEVELOPMENT IN TENSILE LOADING}

A tensile test with intermittent stiffness checks was designed and carried out to explore changes in stiffness with increasing stress and strain. During this test a specimen was loaded to $20 \%$ UTS, unloaded 
to zero stress, at which point a stiffness check was performed. This sequence, consisting of loading to a specific load/stress level, unloading to zero stress and performing a stiffness check, was repeated for load levels of 40,60 , and $80 \%$ UTS. The loading to $80 \%$ UTS, and unloading to zero stress were followed by loading to failure. The monotonic loading portions of the test were displacement controlled with a rate of $0.001 \mathrm{in} . / \mathrm{s}$. Stiffness checks consisted of three load controlled cycles between $100 \mathrm{lb}$ and $500 \mathrm{lb}$ (elastic regime) conducted at a load rate of $40 \mathrm{lb} / \mathrm{s}(240 \mathrm{lb} / \mathrm{min})$. To allow ready comparison of the results with those of the tensile tests reported in Section 3.1.2, the monotonic loading and stiffness checks were conducted in a manner identical to that employed in Experimental Arrangements 1 (see Section 3.1.1).

Six specimens from plaque 42 were subjected to the tensile test with intermittent stiffness checks. Results are presented graphically in Fig. 10, where percent change in stiffness is plotted vs prior maximum load given in terms of \% UTS. It is seen in Fig. 10 that no significant changes in stiffness occur below $30 \%$ UTS. Above this threshold stiffness decreases with increasing prior load, and this dependence may be described with the following linear law:

$$
\Delta \mathrm{E} \times 100 \%=5.5-0.186 \frac{\sigma}{\mathrm{UTS}} \times 100 \% .
$$

\subsection{EFFECT OF RATE}

Thirteen constant strain rate tensile tests to failure were performed to assess the effect of strain rate on tensile properties of the material. The strain rates used in this study were $10^{-6}, 10^{-4}, 10^{-2}$, and $10 \mathrm{~s}^{-1}$. Three tests were conducted at each of the strain rates, with the exception of the strain rate of $10^{-2} \mathrm{~s}^{-1}$, at which four tests were carried out. Specimens cut in the $0^{\circ}$ direction from three different plaques were employed. Test results are summarized in Table 5, where the values of stiffness, ultimate tensile strength, and failure strain obtained at each strain rate are given together with the corresponding averages and standard deviations.

Table 5. Summary of tensile properties obtained at different constant strain rates

\begin{tabular}{lcccc}
\hline \multicolumn{1}{c}{ Specimen \# } & Strain Rate $\left(\mathrm{s}^{-1}\right)$ & Stiffness (Msi) & $\begin{array}{c}\text { UltimateTensile } \\
\text { Strength (ksi) }\end{array}$ & $\begin{array}{c}\text { Failure } \\
\text { Strain }(\%)\end{array}$ \\
\hline P8-0-12 & 10 & 1.10 & 22.1 & 2.20 \\
P8-0-25 & 10 & 1.22 & 24.6 & 2.15 \\
P12-0-7 & 10 & 1.07 & 22.6 & 2.55 \\
Average & & 1.13 & 23.1 & 2.30 \\
St. Deviation & & 0.08 & 1.33 & 0.18 \\
& $10^{-2}$ & & & \\
P8-0-11 & $10^{-2}$ & 1.19 & 21.8 & 2.50 \\
P8-0-27 & $10^{-2}$ & 1.33 & 25.2 & 2.54 \\
P12-0-5 & $10^{-2}$ & 1.15 & 21.3 & 2.61 \\
P12-0-6 & & 1.17 & 22.5 & 2.88 \\
Average & & 1.21 & 22.7 & 2.63 \\
St. Deviation & & 0.08 & 1.73 & 0.17 \\
& & & & \\
P8-0-24 & $10^{-4}$ & 1.36 & 21.6 & 2.22 \\
P12-0-2 & $10^{-4}$ & 1.23 & 19.9 & 2.34 \\
P15-0-2 & $10^{-4}$ & 1.18 & 18.7 & 2.20 \\
Average & & 1.26 & 20.1 & 2.25 \\
St. Deviation & & 0.09 & 1.44 & 0.08 \\
& & & & \\
P8-0-26 & $10^{-6}$ & 1.14 & 17.4 & 1.93 \\
P12-0-4 & $10^{-6}$ & 1.02 & 16.3 & 2.35 \\
P15-0-5 & $10^{-5}$ & 1.12 & 16.1 & 2.13 \\
Average & & 1.09 & 16.6 & 2.14 \\
St. Deviation & & 0.07 & 0.71 & 0.21 \\
\hline
\end{tabular}


Results are also graphically presented in Figs. 11-14. The plot of stiffness vs strain rate in Fig. 11 shows an increase in stiffness as the strain rate increases from $10^{-6}$ to $10^{-4} \mathrm{~s}^{-1}$ followed by decreasing stiffness as the strain rate continues to increase from $10^{-4}$ to $10 \mathrm{~s}^{-1}$. This trend is observed for each plaque. However, it should be noted that the changes in stiffness are small and, in fact, comparable to standard deviations calculated along with the average stiffness for each strain rate. The variations in failure strain with strain rate shown in Fig. 12 are likewise fairly small, but for each plaque a distinct tendency may be observed where failure strain increases as the strain rate increases from $10^{-6}$ to $10^{-2} \mathrm{~s}^{-1}$ and decreases as the strain rate continues to increase to $10 \mathrm{~s}^{-1}$. Conversely, the effect of strain rate on ultimate tensile strength illustrated in Fig. 13 appears to be more pronounced. As the strain rate increases from $10^{-6}$ to $10 \mathrm{~s}^{-1}$ the average ultimate tensile strength increases by about $39 \%$. Such a considerable change in the ultimate tensile strength indicates that this property is significantly affected by strain rate. Stress-strain curves obtained at four different strain rates and combined in Fig. 14 indicate that while there is a slight strain rate sensitivity, it decreases with increasing strain rate.

Significant specimen to specimen variations in stiffness for this material were reported in Section 3.1.2 above. To ensure that the rate effects presented here were indeed due to strain rate change and not to the initial differences in stiffness, the values of stress and ultimate tensile strength for each individual specimen were normalized by the stiffness of that particular specimen. Normalized results presented in Figs. 15 and 16 support the conclusions made above. 


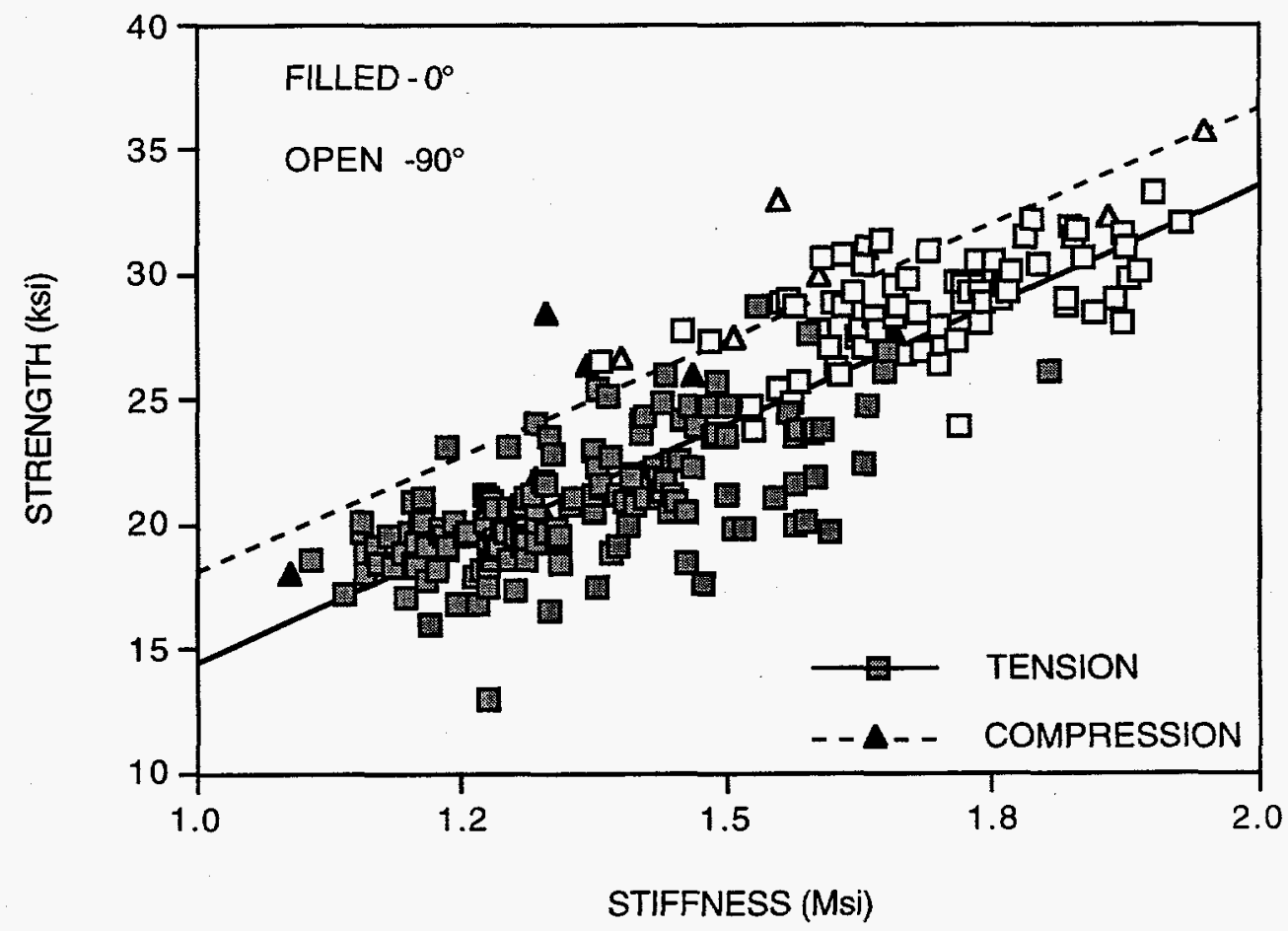

Fig. 2. Strength vs stiffness.

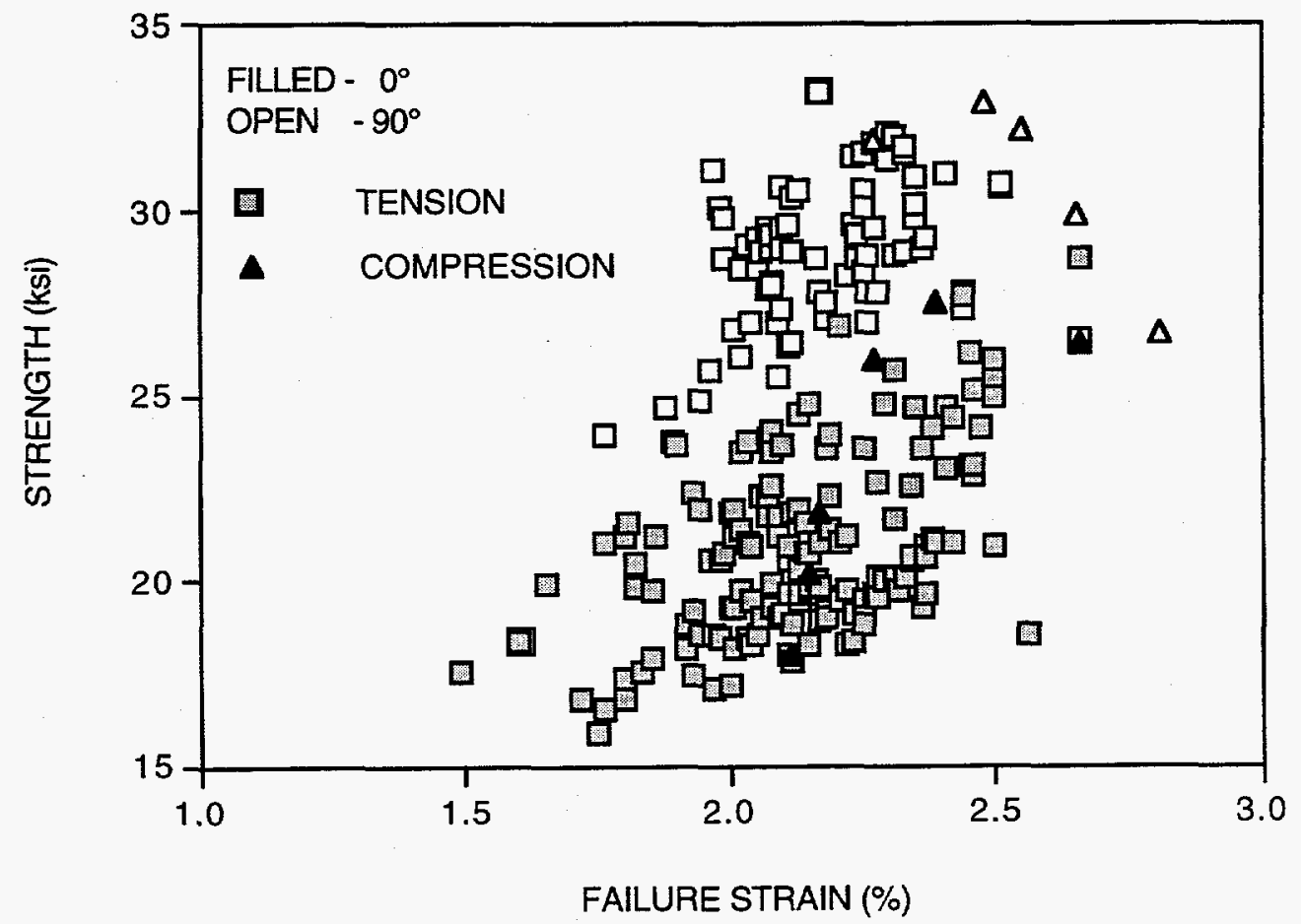

Fig. 3. Strength vs failure strain. 


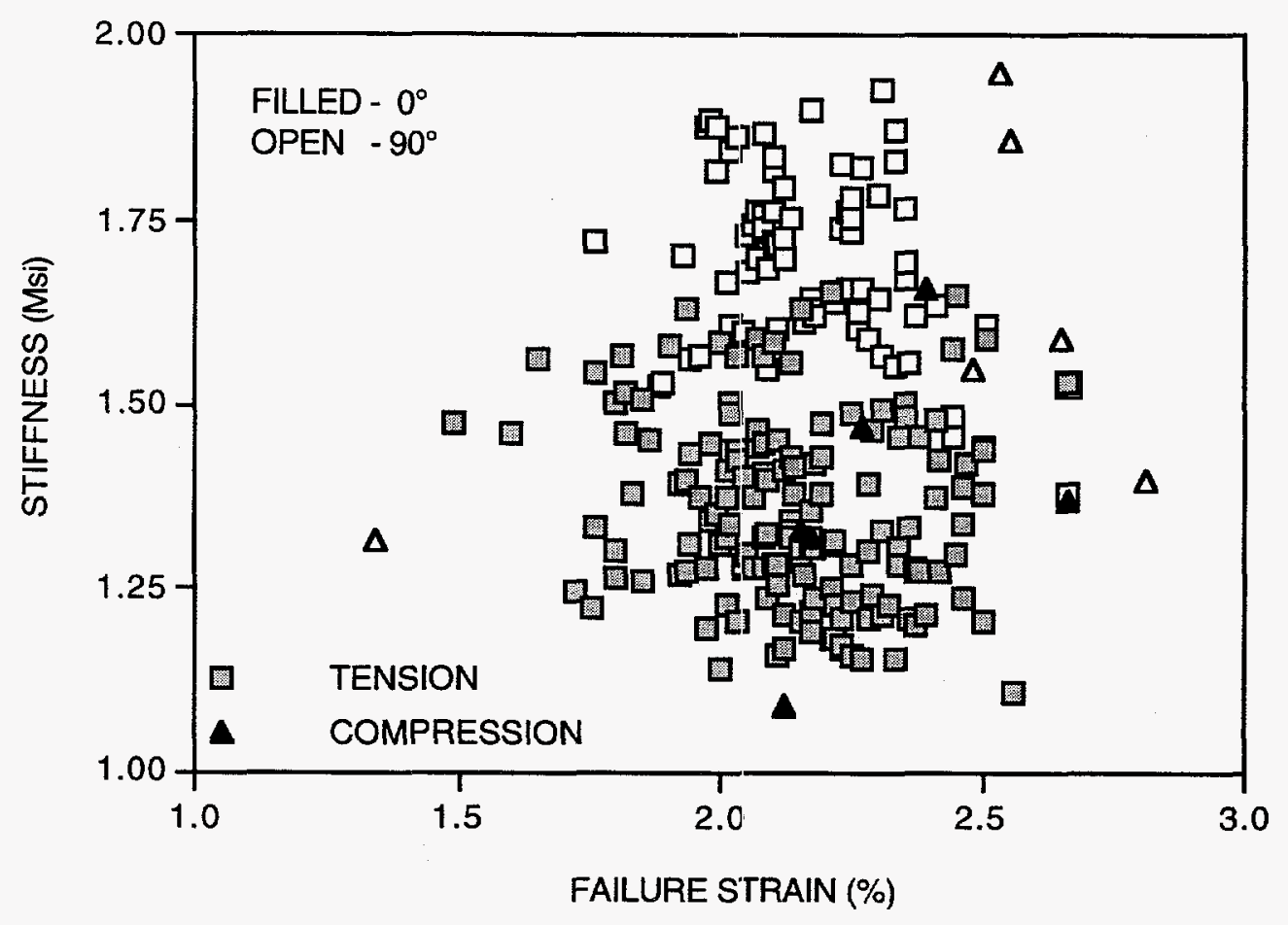

Fig. 4. Stiffness vs failure strain.

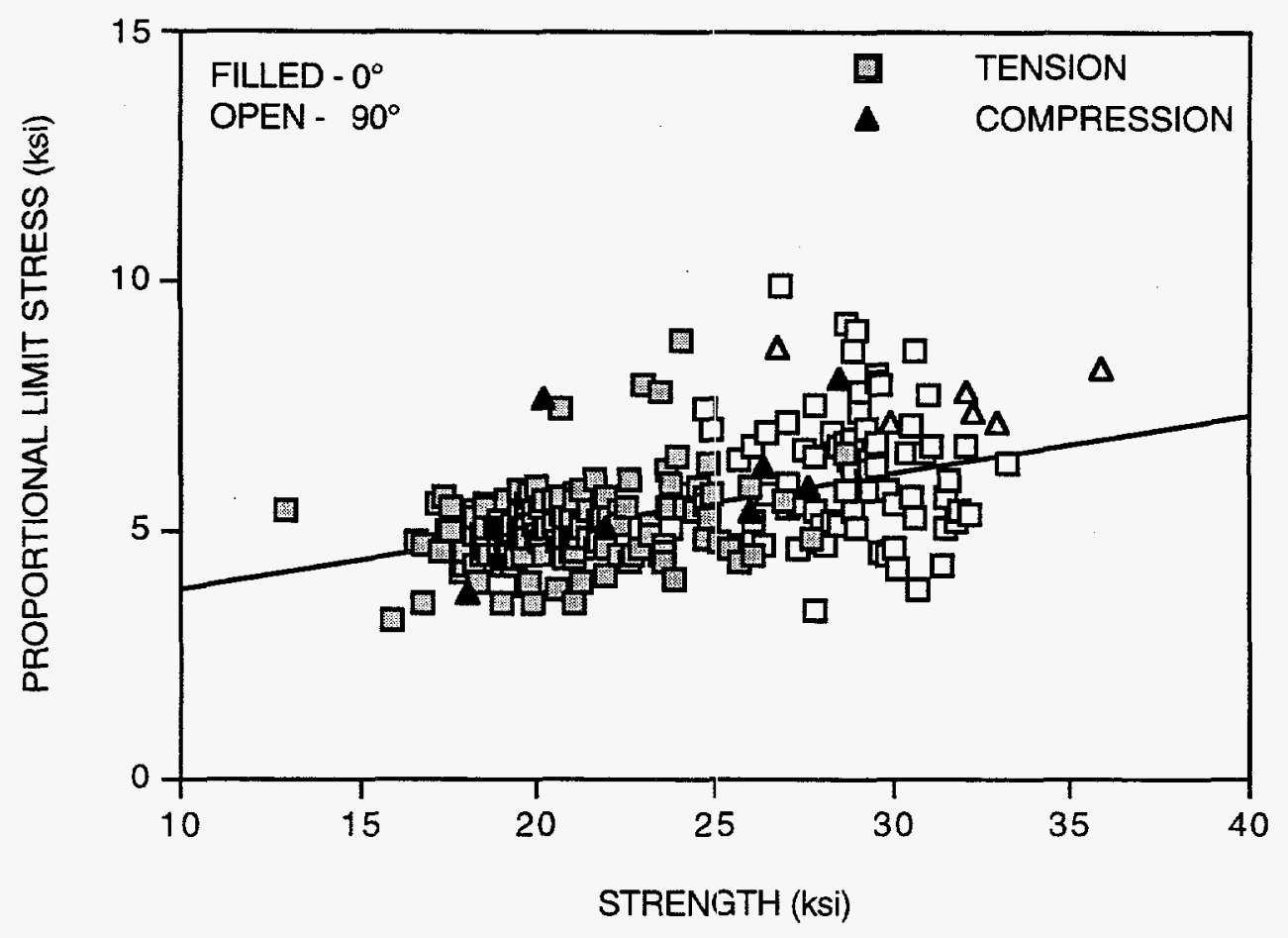

Fig. 5. Proportional limit stress vs strength. 


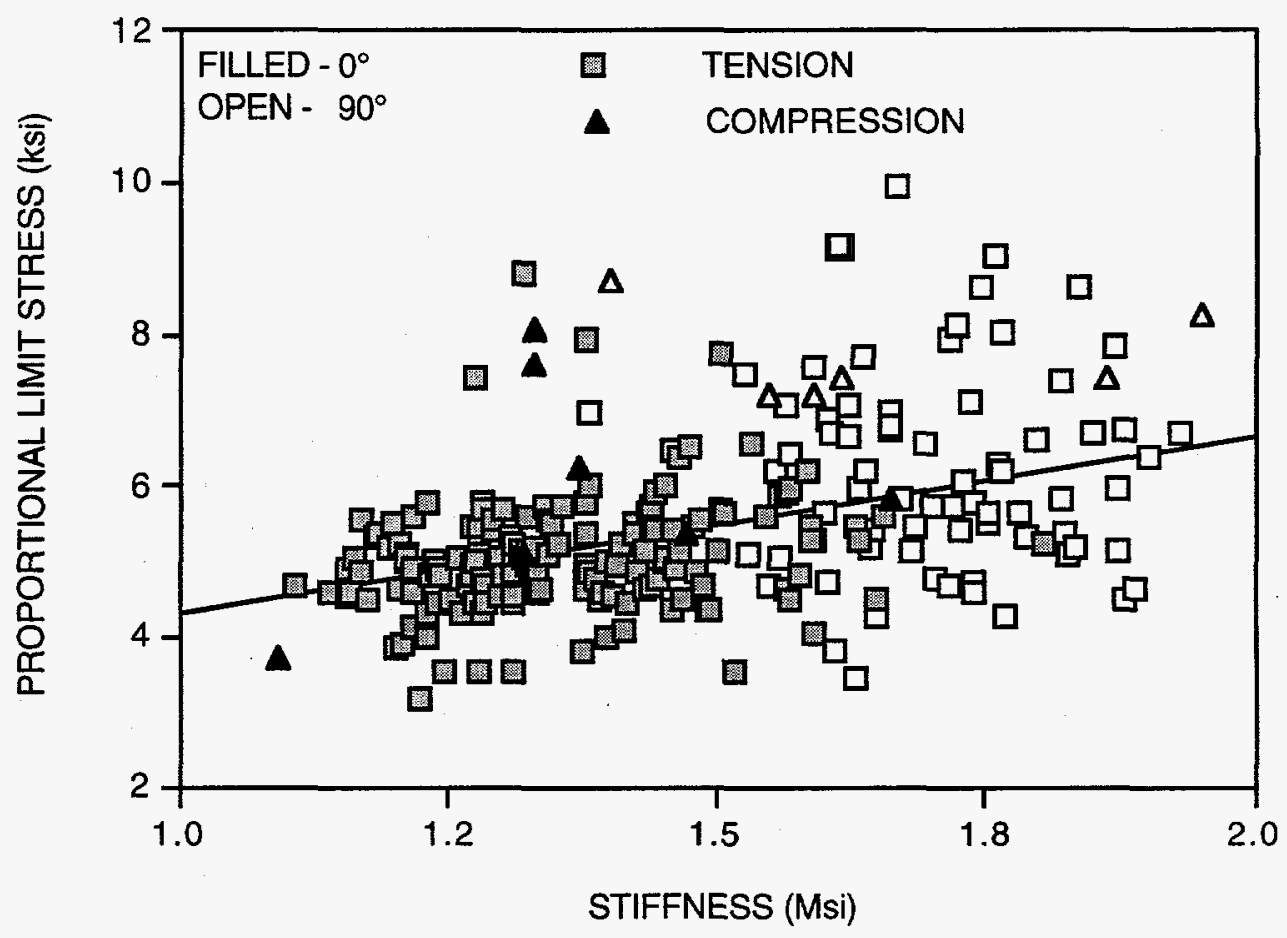

Fig. 6. Proportional limit stress vs stiffness.

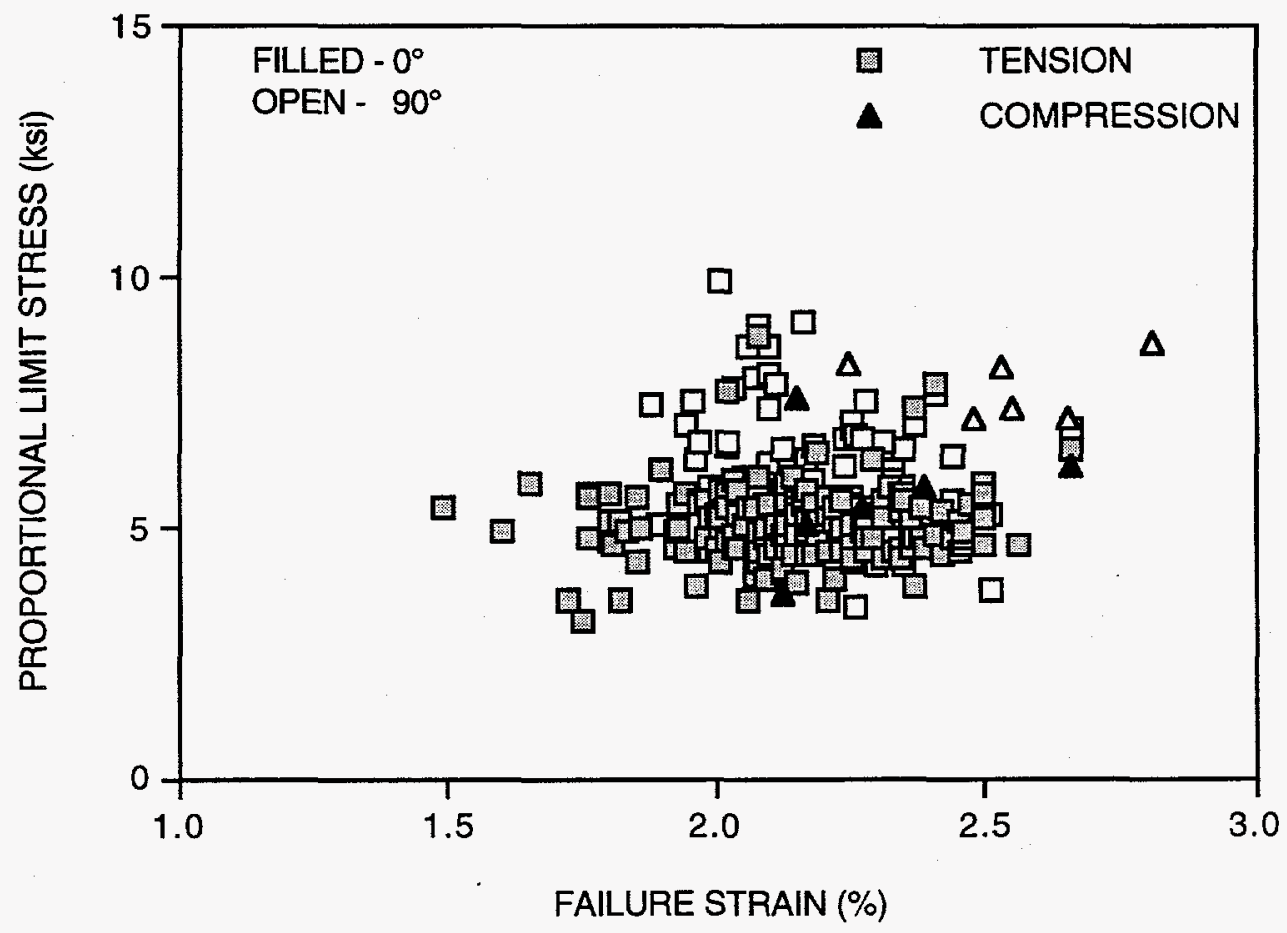

Fig. 7. Proportional limit stress vs failure strain. 


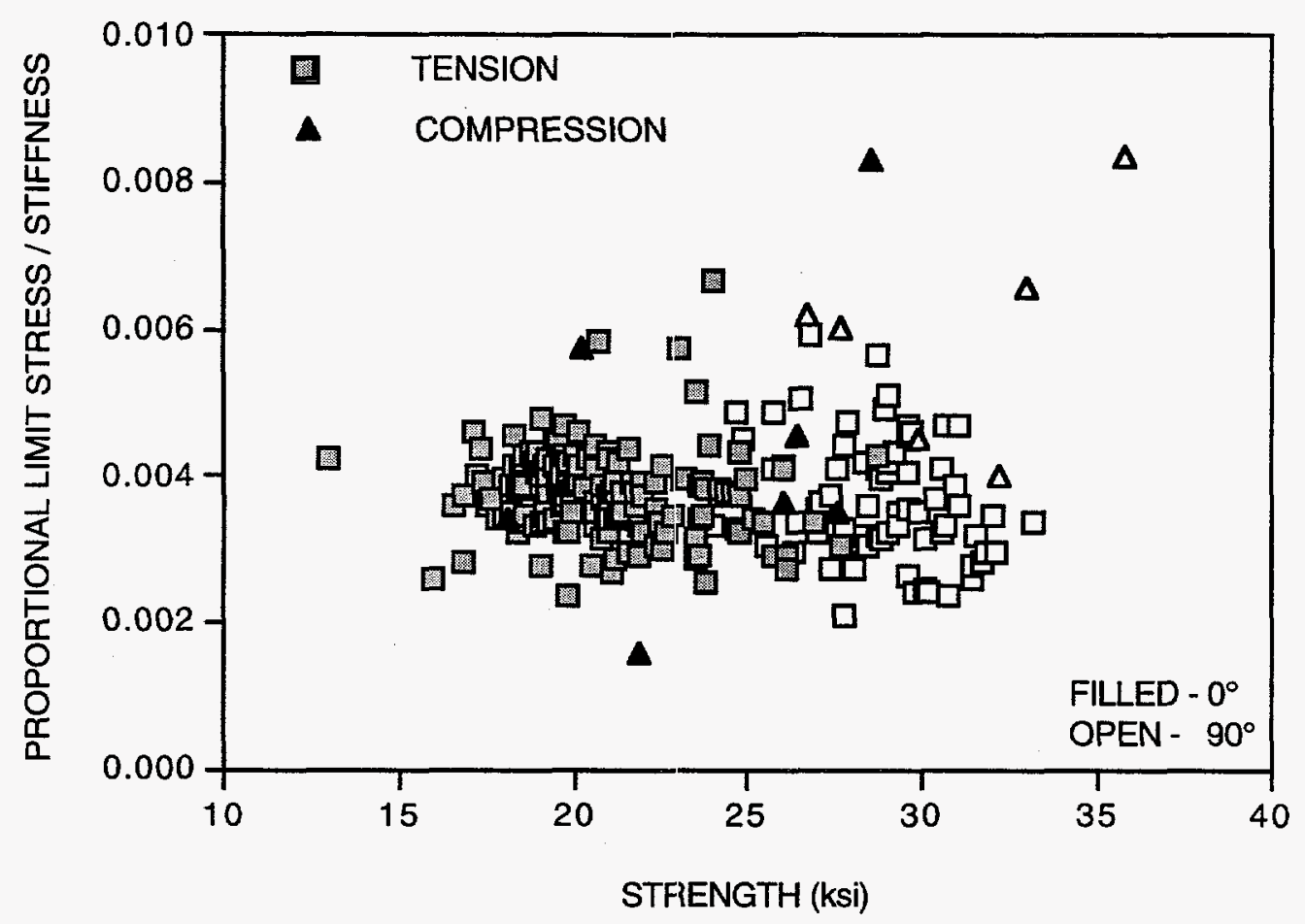

Fig. 8. Proportional limit stress normalized by stiffness vs strength.

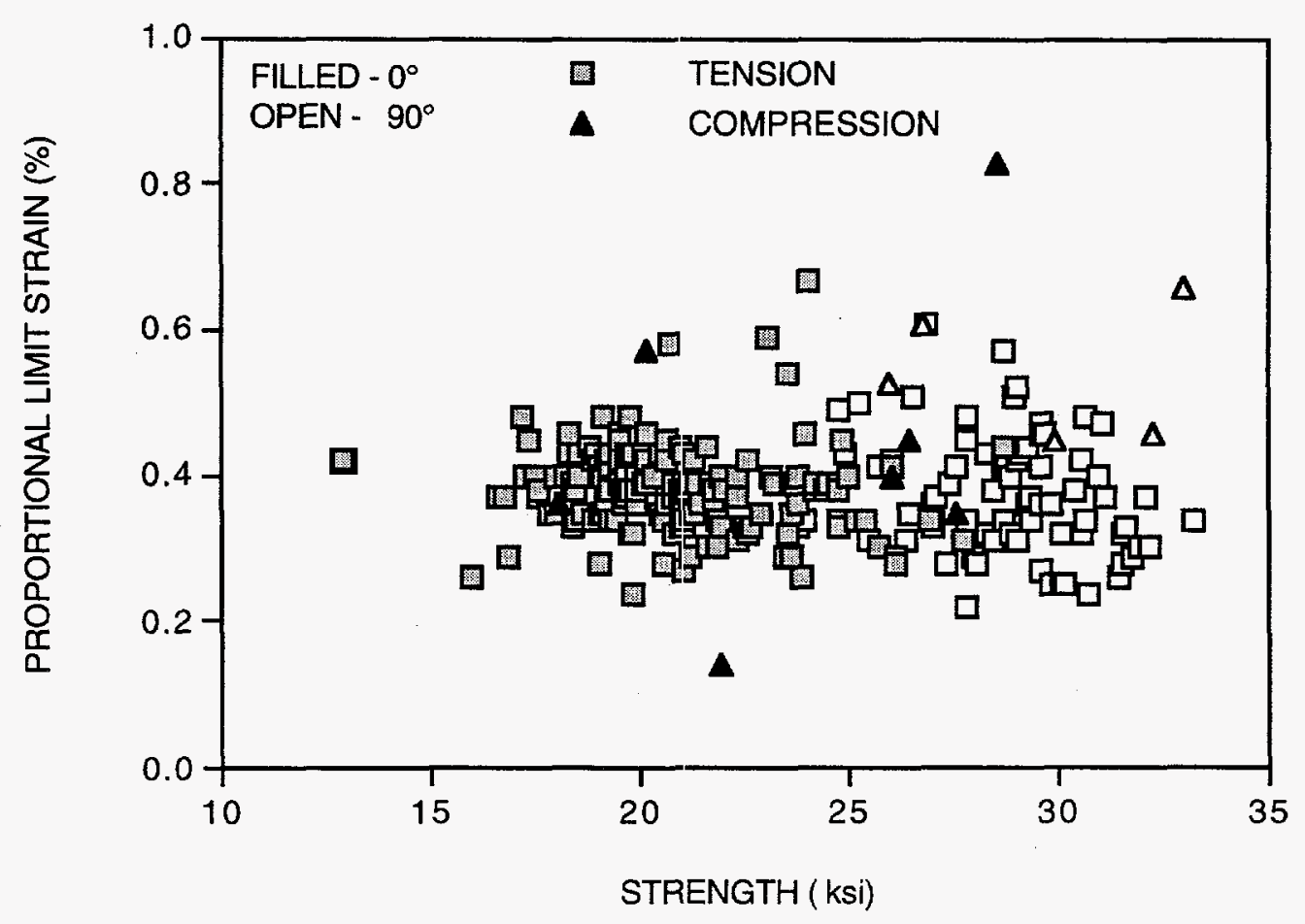

Fig. 9. Proportional limit strain vs strength. 


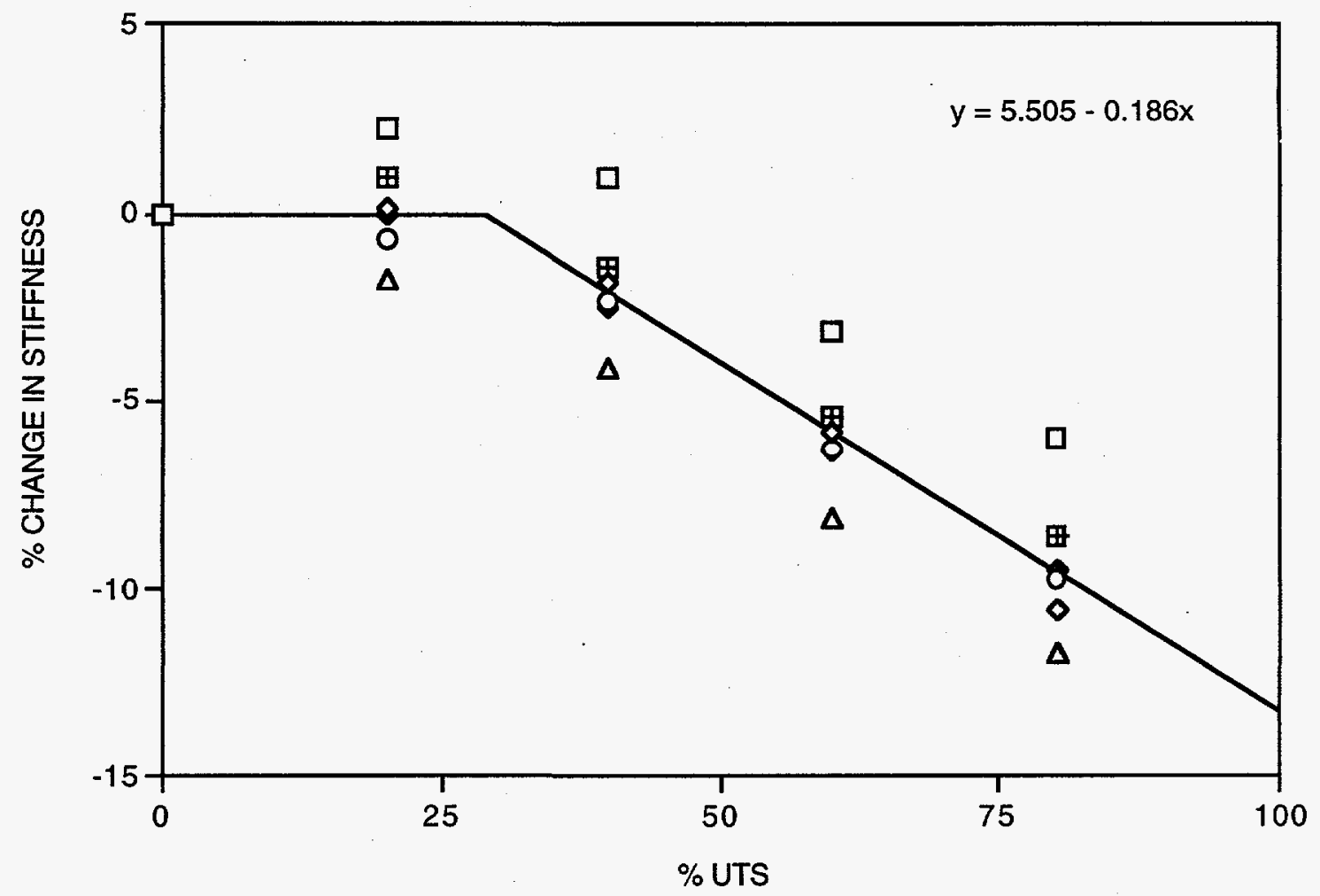

Fig. 10. Percent change in stiffness vs prior maximum load given in terms of \%UTS.

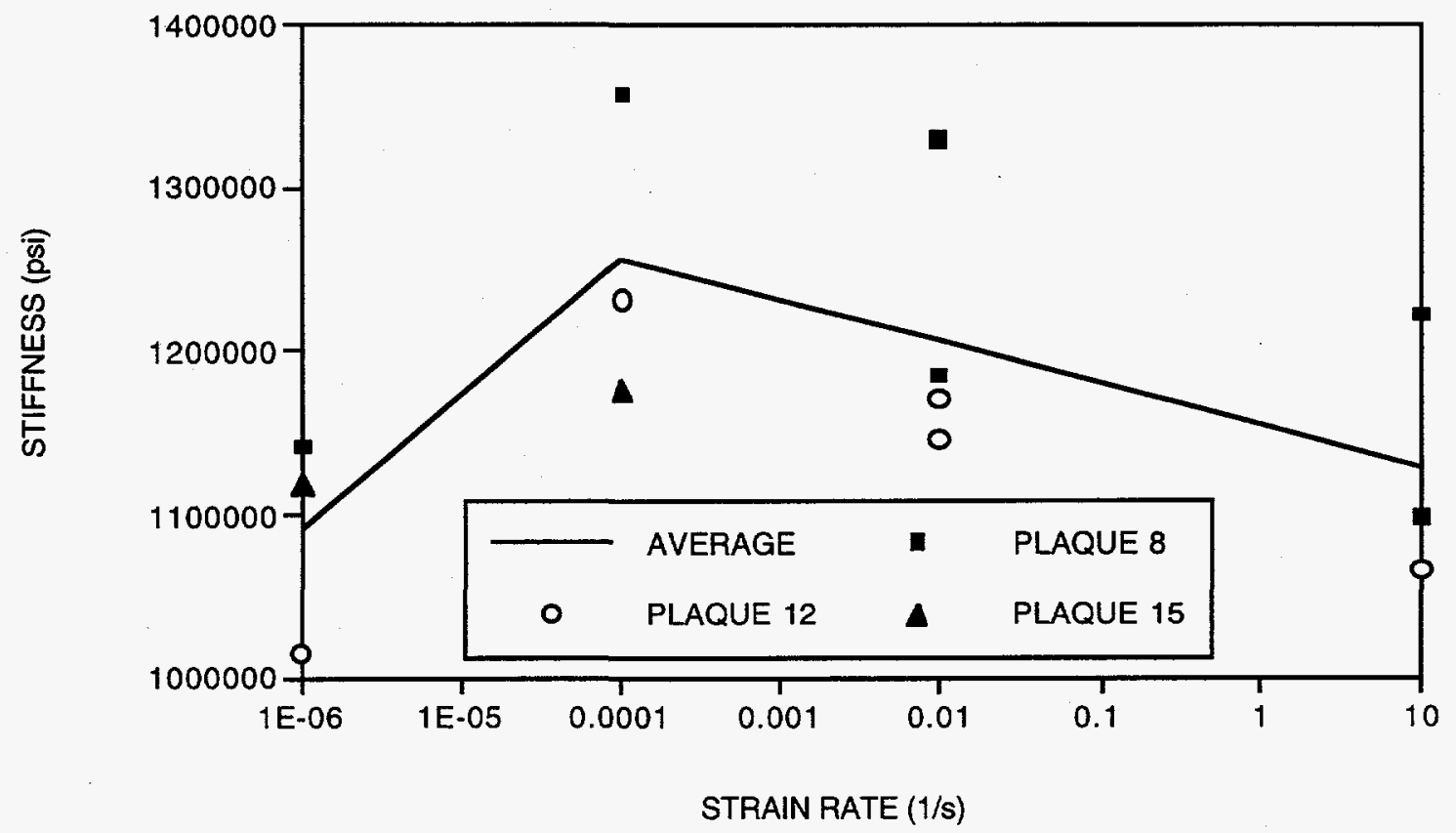

Fig. 11. Variation in stiffness with strain rate. 


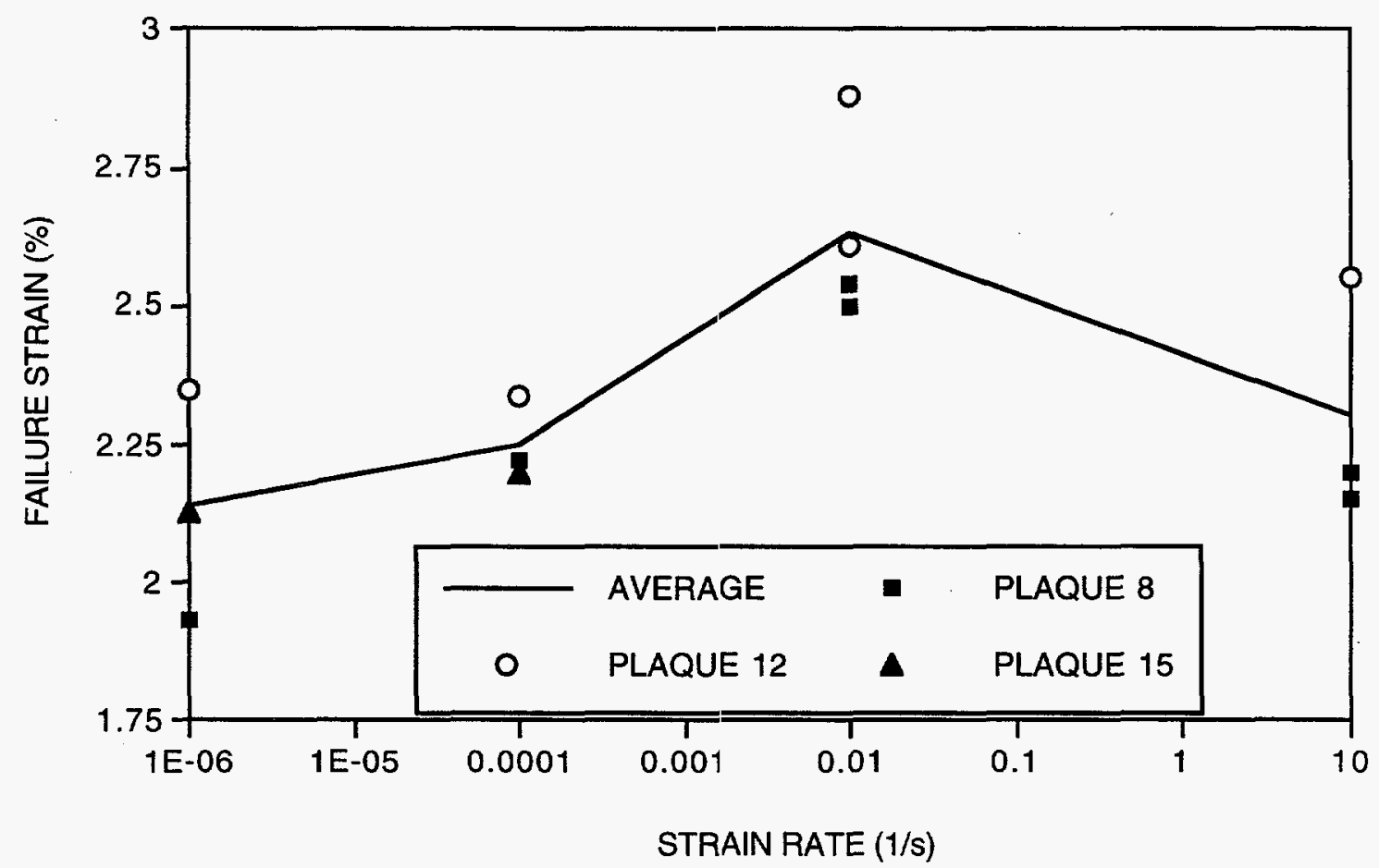

Fig. 12. Variation in failure strain with strain rate.

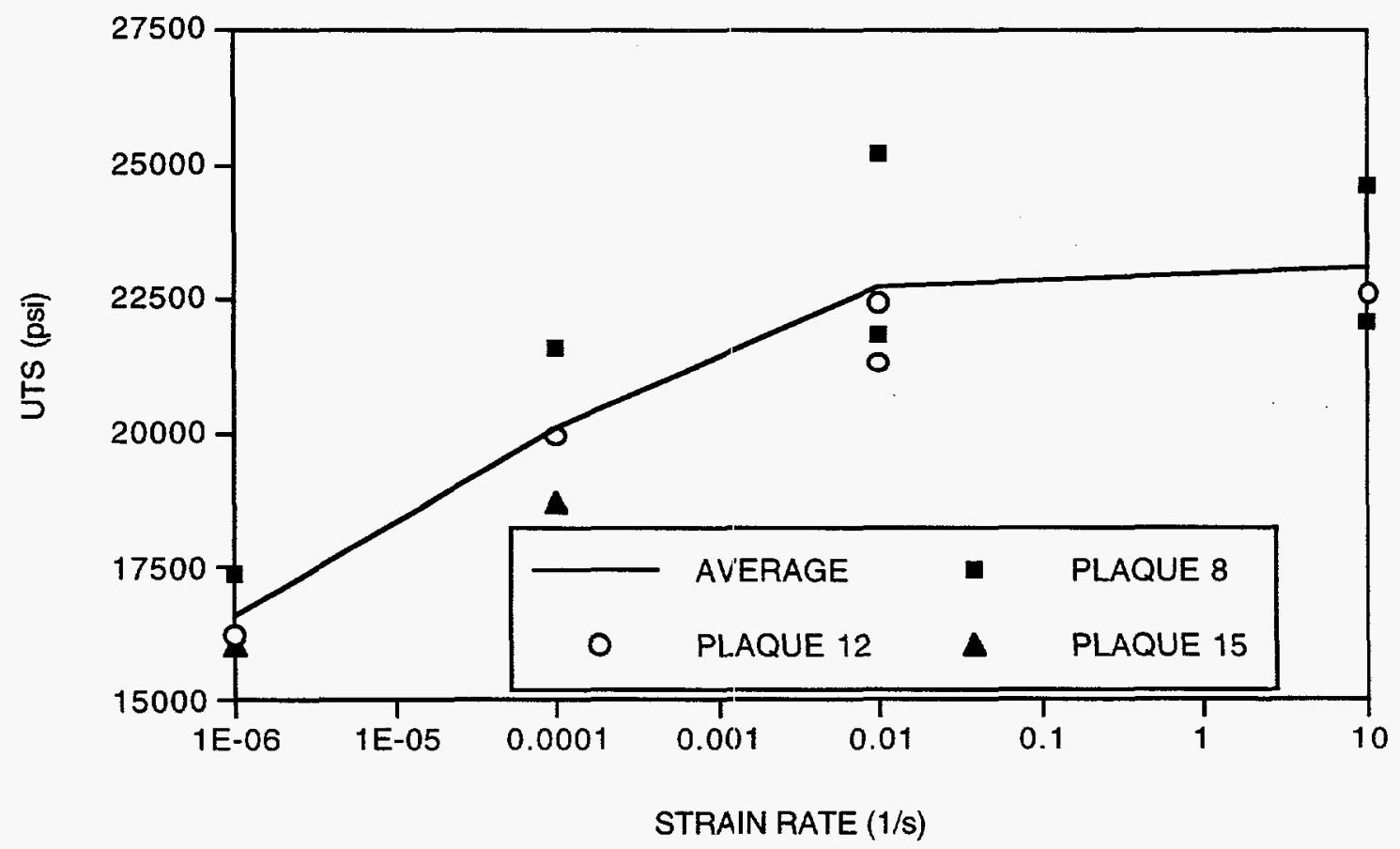

Fig. 13. Effect of strain rate on ultimate tensile strength. 


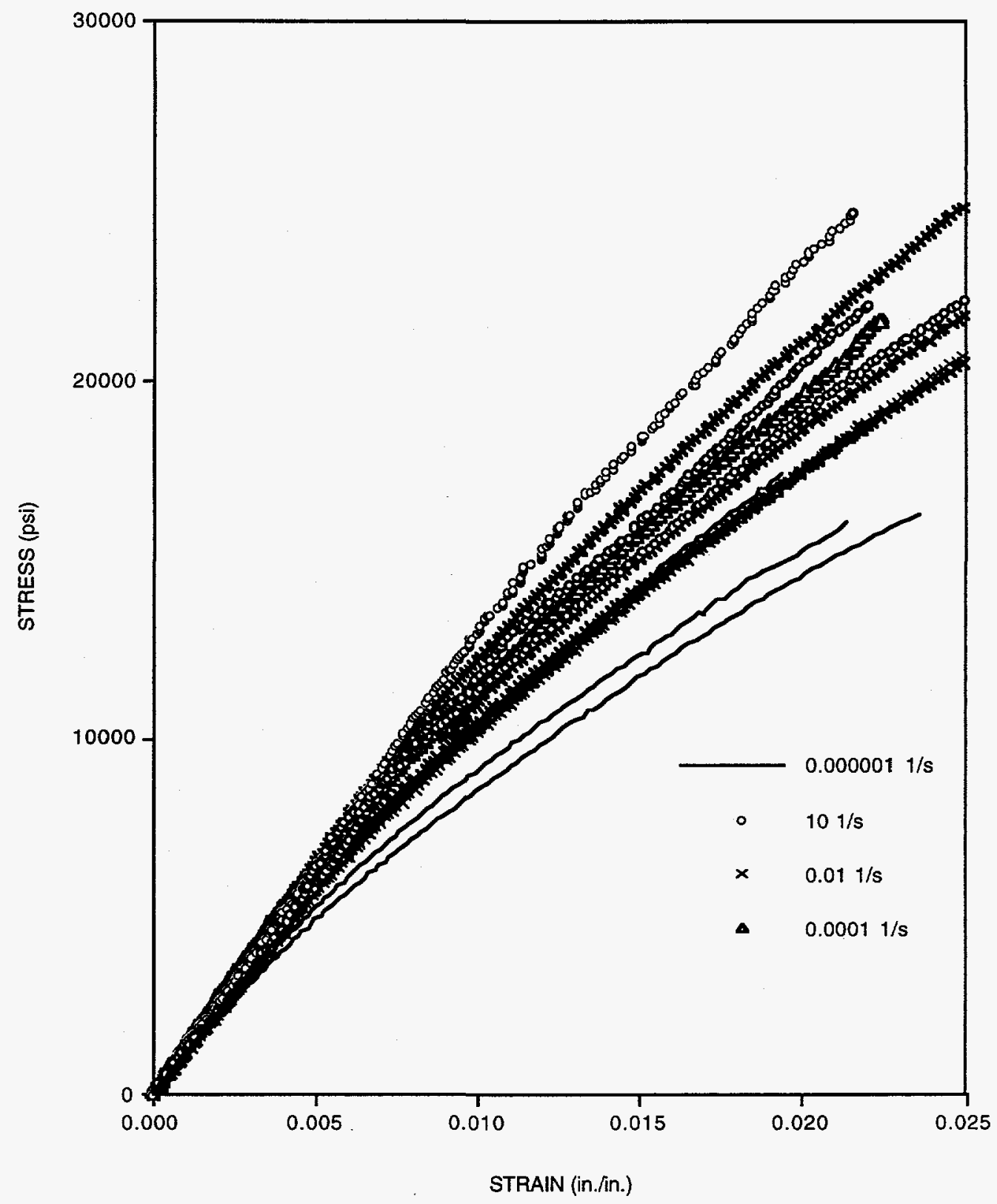

Fig. 14. Stress-strain curves obtained at the strain rates of $10^{-6}, 10^{-4}, 10^{-2}$, and $10 \mathrm{~s}^{-1}$. 


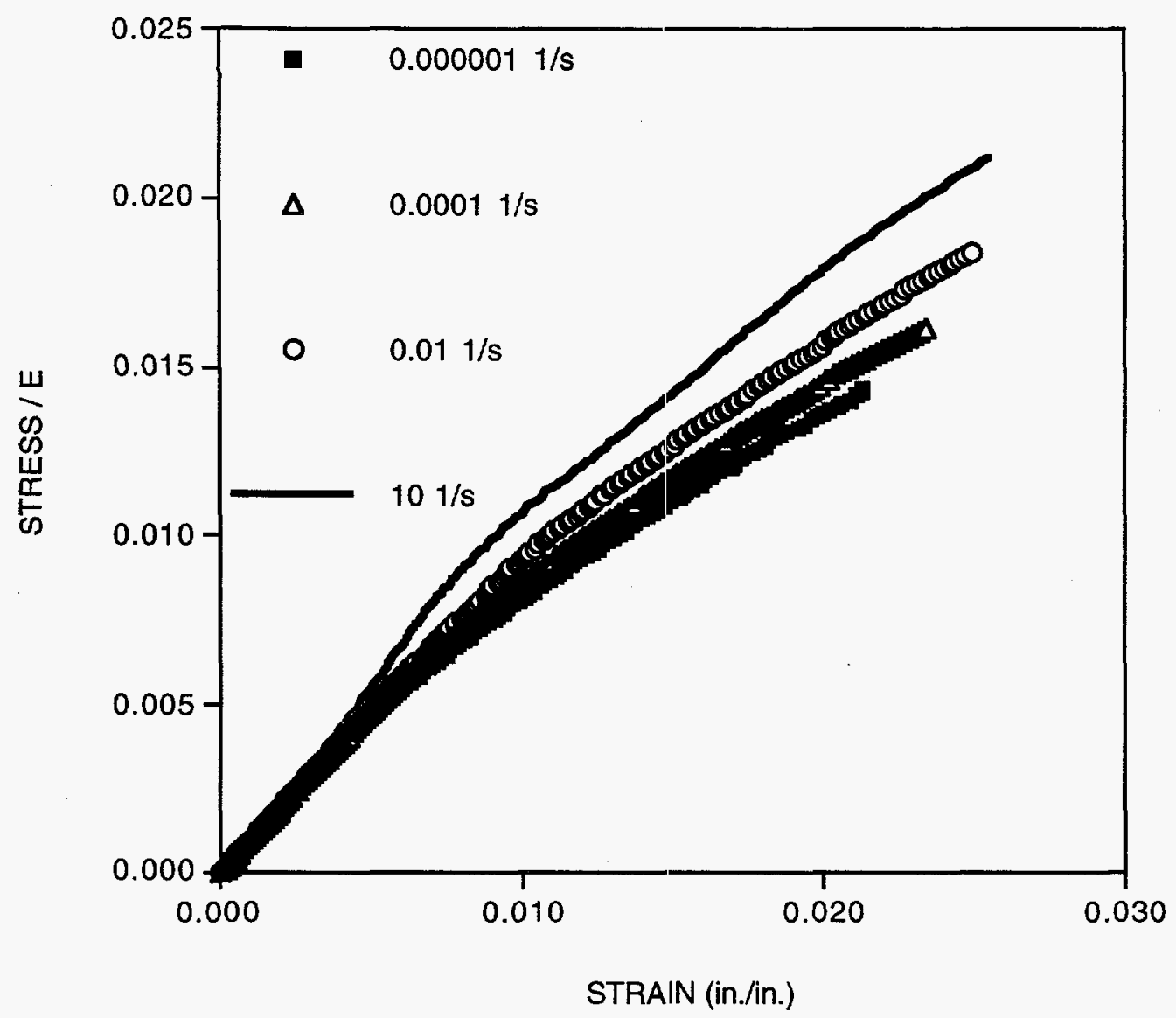

Fig. 15. Normalized stress-strain curves at the strain rates of $10^{-6}, 10^{-4}, 10^{-2}$, and $10 \mathrm{~s}^{-1}$.

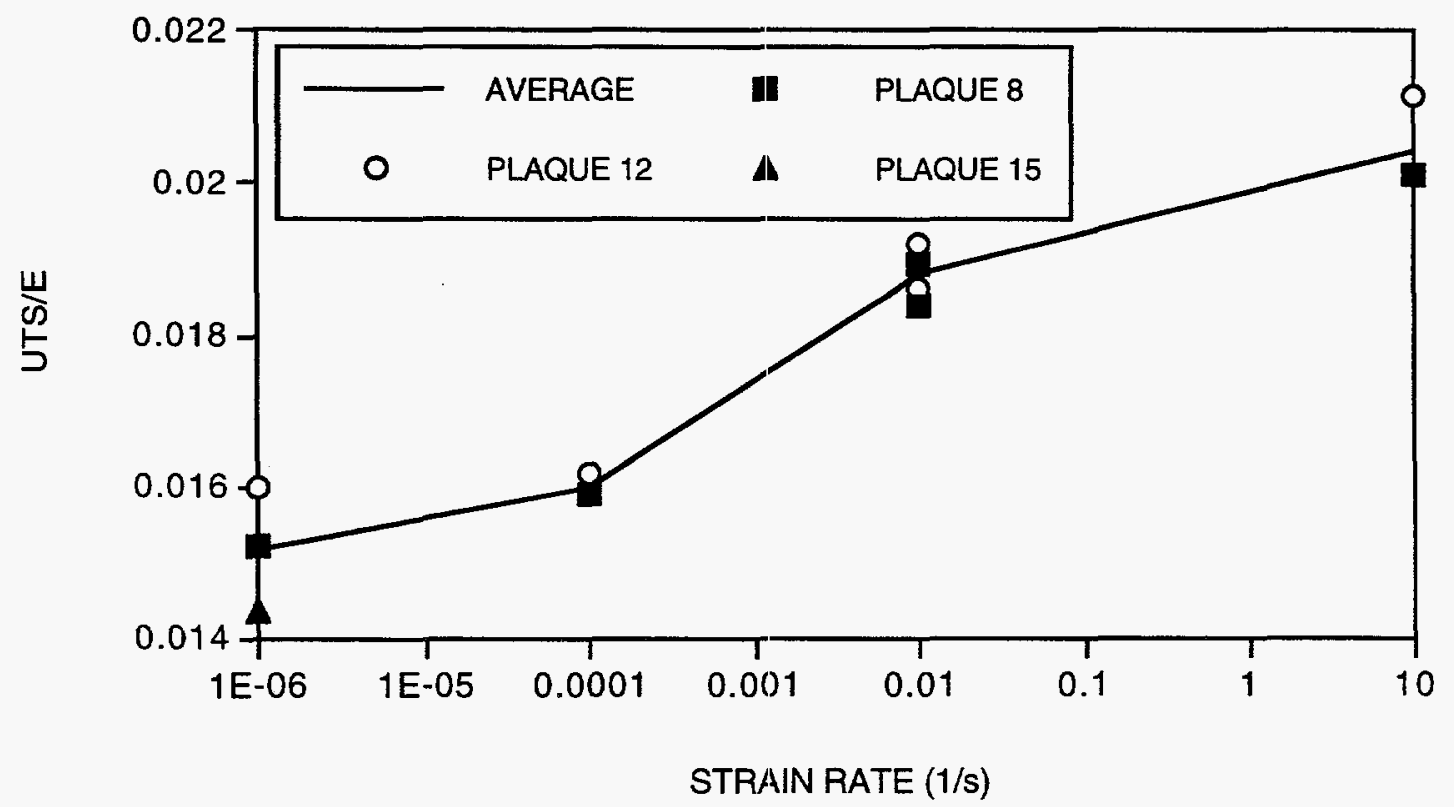

Fig. 16. Variation in ultimate tensile strengthı normalized by stiffness with strain rate. 


\section{COMPRESSIVE BEHAVIOR}

\subsection{BASELINE TESTS}

\subsubsection{Experimental Arrangements}

All tests were performed at room temperature in an air environment. The servocontrolled MTS axial-torsion mechanical testing machine together with the MTS digital TestStar Materials Testing Workstation employed in the tensile tests reported above was used for computerized compression tests and data acquisition. Specimens were mounted in an IITRI Compression Test Fixture as outlined in ASTM Standard D 3410. Compressive tests to failure were conducted in displacement control at a rate of 0.001 in./s. Strain measurement was accomplished with two strain gages mounted in the gage section of the specimen, one strain gage on each side of the specimen. In most tests, the two strain gages produced strain measurements that were approximately the same. The two strain measurements were averaged and the average strains were used for reporting data. The data acquisition intervals were established on the basis of load, i.e. a data point was recorded whenever the load changed by $10 \mathrm{lb}$. The digitized test data were stored on both hard and floppy disks. Compressive stiffness was calculated using linear regression for the initial stress-strain slope from 800 to 4000 psi.

\subsubsection{Summary of Compressive Tests}

Twelve specimens were subjected to compression tests at room temperature in an air environment. Seven specimens were cut in the $0^{\circ}$ direction from plaques P10 and P37; five specimens were cut from plaque $\mathrm{P} 37$ in the $90^{\circ}$ direction. Test results are summarized below.

Table 6. Summary of compressive tests conducted at room temperature in an air environment

\begin{tabular}{|c|c|c|c|c|c|}
\hline $\begin{array}{l}\text { Specimen } \\
\text { Number }\end{array}$ & $\begin{array}{l}\text { Compressive } \\
\text { Stiffness } \\
\text { (Msi) }\end{array}$ & $\begin{array}{c}\text { Ultimate } \\
\text { Compressive } \\
\text { Strength (ksi) }\end{array}$ & $\begin{array}{c}\text { Failure Strain } \\
(\%)\end{array}$ & $\begin{array}{l}\text { Proportional } \\
\text { Limit Stress } \\
\quad \text { (ksi) }\end{array}$ & $\begin{array}{l}\text { Proportional } \\
\text { Limit Strain } \\
\text { (\%) }\end{array}$ \\
\hline $\begin{array}{l}\text { P10-0-8 } \\
\text { P10-0-10 } \\
\text { P10-0-12 } \\
\text { P10-0-11 } \\
\text { P37-0-1 } \\
\text { P37-0-5 } \\
\text { P37-0-9 }\end{array}$ & $\begin{array}{l}1.47 \\
1.32 \\
1.09 \\
1.33 \\
1.33 \\
1.66 \\
1.37\end{array}$ & $\begin{array}{c}0^{\circ}-\text { direction } \\
-26.0 \\
-21.9 \\
-18.1 \\
-20.2 \\
-28.5 \\
-27.6 \\
-26.4\end{array}$ & $\begin{array}{l}-2.27 \\
-2.17 \\
-2.12 \\
-2.15 \\
-3.11 \\
-2.39 \\
-2.66\end{array}$ & $\begin{array}{l}-5.39 \\
-5.08 \\
-3.71 \\
-7.63 \\
-8.07 \\
-5.85 \\
-6.26\end{array}$ & $\begin{array}{l}-0.40 \\
-0.14 \\
-0.36 \\
-0.57 \\
-0.83 \\
-0.35 \\
-0.45\end{array}$ \\
\hline $\begin{array}{l}\text { Average } \\
\text { St. Deviation } \\
\text { Coefficient of } \\
\quad \text { Variation (\%) }\end{array}$ & $\begin{array}{l}1.37 \\
0.17 \\
12.4\end{array}$ & $\begin{array}{l}-24.1 \\
4.03 \\
16.8\end{array}$ & $\begin{array}{l}-2.41 \\
0.36 \\
14.9\end{array}$ & $\begin{array}{l}-6.00 \\
1.50 \\
25.0\end{array}$ & $\begin{array}{r}-0.44 \\
0.21 \\
47.7\end{array}$ \\
\hline $\begin{array}{l}\text { P37-90-1 } \\
\text { P37-90-5 } \\
\text { P37-90-5A } \\
\text { P37-90-10A } \\
\text { P37-90-8 }\end{array}$ & $\begin{array}{l}1.40 \\
1.55 \\
1.86 \\
1.95 \\
1.59\end{array}$ & $\begin{array}{c}90^{\circ} \text {-direction } \\
-26.8 \\
-33.0 \\
-32.3 \\
-35.8 \\
-29.9\end{array}$ & $\begin{array}{l}-2.81 \\
-2.48 \\
-2.55 \\
-2.53 \\
-2.65\end{array}$ & $\begin{array}{l}-8.70 \\
-7.21 \\
-7.42 \\
-8.26 \\
-7.20\end{array}$ & $\begin{array}{l}-0.61 \\
-0.66 \\
-0.46 \\
-0.84 \\
-0.45\end{array}$ \\
\hline $\begin{array}{l}\text { Average } \\
\text { St. Deviation } \\
\text { Coefficient of } \\
\quad \text { Variation (\%) }\end{array}$ & $\begin{array}{l}1.67 \\
0.23 \\
13.8\end{array}$ & $\begin{array}{l}-31.5 \\
3.40 \\
10.8\end{array}$ & $\begin{array}{l}-2.60 \\
0.13 \\
5.00\end{array}$ & $\begin{array}{l}-7.76 \\
0.68 \\
8.76\end{array}$ & $\begin{array}{r}-0.60 \\
0.16 \\
26.7\end{array}$ \\
\hline
\end{tabular}


It is noteworthy that all compression specimens failed in the middle of the gage section with the failure surfaces oriented at a $45^{\circ}$ angle to the specimen axis. Results in Table 6 demonstrate that, as in the case of tension, the $90^{\circ}$ direction is the "strong" direction, the $90^{\circ}$ direction specimens exhibited higher compressive stiffness and ultimate compressive strength (UCS) values than the $0^{\circ}$ direction specimens. Average compressive stiffness $\mathrm{E}_{C}$ for the $90^{\circ}$ direction is $22 \%$ higher than the average $\mathrm{E}_{\mathrm{C}}$ obtained for the $0^{\circ}$ direction. This compares very well with the tensile results, where the average $\mathrm{E}$ obtained for the $90^{\circ}$ direction was $23 \%$ higher than the average $\mathrm{E}$ for the $0^{\circ}$ direction. Average ultimate compressive strength (UCS) measured for the $90^{\circ}$ direction is $31 \%$ higher than the average UCS obtained for the $0^{\circ}$ direction, which again compares very well with the tensile results where the UTS values for the $90^{\circ}$ direction were $34 \%$ higher than those for the $0^{\circ}$ direction. However, it should be noted that the average compressive failure strains obtained for the two directions are further apart than those obtained in tensile tests.

It is seen in Table 6 that similar values of the coefficients of variation were obtained for all compressive properties in both the $0^{\circ}$ and the $90^{\circ}$ directions. The same observation was made earlier regarding tensile properties. For the $0^{\circ}$ direction, compressive properties exhibited slightly higher values of the coefficient of variation (i.e. higher amount of scatter) than the tensile properties. For the $90^{\circ}$ direction, however, only compressive stiffness showed more scatter than the tensile stiffness; UTS and tensile failure strain exhibited more scatter than the corresponding cornpressive properties.

\subsection{BEHAVIORAL TRENDS-CORRELATIONS BETWEEN COMPRESSIVE PROPERTIES}

For a ready comparison, average compressive and tensile properties are recapitulated below:

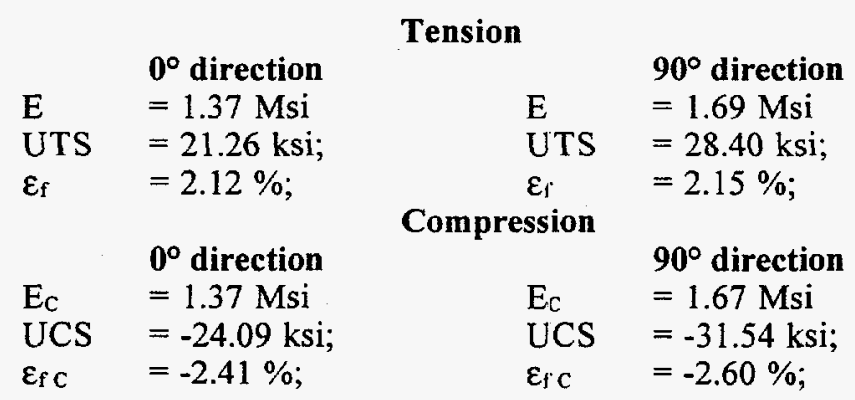

It can be seen that the stress-strain behavior is symmetric, i.e. compressive and tensile properties are comparable.

To establish possible correlations, compressive properties were assessed in a manner similar to that used for assessing tensile properties in Section 3.2. Ultimate compressive strength, compressive stiffness, and failure strain were cross-plotted in Figs. 2-4. To facilitate comparison with tensile behavior, plots in Figs. 2-4 include tensile results and employ absolute values of UCS and compressive failure strain. It is seen that compressive and tensile properties represent a consistent set of data. The plot of strength vs stiffness in Fig. 2 reveals that the tensile and compressive sets of data can be approximated with straight lines of the same slope, i.e. linear laws with the same coefficients can be used to describe dependence of strength on stiffness for both tension and compression. Plots of strength and stiffness vs failure strain in Figs. 3 and 4, respectively, show that in both tension and compression strength and stiffness are independent of the failure strain. It can be further added that in compression, as in tension, failure strain remains independent of strength and/or stifiness.

Based on the compression test results compressive proportional limits were established as follows:

$$
\begin{aligned}
& \mathbf{0}^{\circ}-\text { direction } \\
& \mathrm{UCS}_{\mathrm{avg}}=-24.09 \mathrm{ksi} ; \\
& \sigma_{\mathrm{PLC} \text { avg }}=-6.00 \mathrm{ksi} \\
& \varepsilon_{\mathrm{FC} \text { avg }}=-2.41 \% \\
& \varepsilon_{\mathrm{PLC} \text { avg }}=-0.44 \% .
\end{aligned}
$$

$$
\begin{aligned}
& \mathbf{9 0}^{\circ} \text { - direction } \\
& \mathrm{UCS}_{\text {avg }}=-31.54 \mathrm{ksi} ; \\
& \sigma_{\mathrm{PLC} \text { avg }}=-7.76 \mathrm{ksi} ; \\
& \varepsilon_{\mathrm{FC} \mathrm{avg}}=-2.60 \% ; \\
& \varepsilon_{\mathrm{PLC} \text { avg }}=-0.60 \% ;
\end{aligned}
$$

The proportional limit stress constitutes $24.9 \%$ and $24.6 \%$ of the UCS for the $0^{\circ}$ and $90^{\circ}$ directions, respectively. Proportional limit strain constitutes $18.2 \%$ and $23.1 \%$ of the failure strain for the $0^{\circ}$ and $90^{\circ}$ directions, respectively. These values compare reasonably well with those obtained in tensile tests. 
Compressive proportional limit stress and strain values were assessed in the same manner as the tensile ones. Proportional limit stresses and strains were plotted vs UCS, stiffness, and failure strain. Proportional limit stresses were normalized by stiffness and plotted vs UCS. Results are presented in Figs. 5-9 together with the tensile results. Graphs in Figs. 5-7, where proportional limit stress is plotted vs strength, stiffness, and failure strain, respectively, show that observations made in Section 3.3 above regarding proportional limit stress behavior in tension apply in compression as well. Compressive results in Figs. 5-7 merge well with the data obtained in tension. Thus it may be concluded that in compression, as well as in tension, proportional limit stress shows a slight increase with strength and stiffness and is independent of the failure strain. Similar observations can be made with respect to the proportional limit stress normalized by stiffness, $\mathrm{s}_{\mathrm{PL}} / \mathrm{E}$. It is seen in Fig. 8 that $\mathrm{s}_{\mathrm{PL}} / \mathrm{E}$ remains fairly independent of strength in both tension and compression. Proportional limit strain behavior is illustrated in Fig. 9. Once more compressive results are close to those obtained in tension, thus suggesting the same conclusions. In compression as well as in tension proportional limit strain appears to be independent of strength, stiffness, or of failure strain.

\subsection{EFFECT OF RATE}

Six constant rate tests were carried out in compression to evaluate the effect of loading rate. All tests were load (stress) controlled. An equivalent strain rate was calculated for each load rate. The load rates were (with equivalent strain rate given in parentheses) $7.5 \mathrm{lb} / \mathrm{min}\left(10-6 \mathrm{~s}^{-1}\right), 2330 \mathrm{lb} / \mathrm{min}\left(3 \times 10-4 \mathrm{~s}^{-1}\right)$, and $13,980 \mathrm{lb} / \mathrm{min}\left(2 \times 10-3 \mathrm{~s}^{-1}\right)$. Three tests were conducted with the rate of $7.5 \mathrm{lb} / \mathrm{min}$, two with the rate of $13,980 \mathrm{lb} / \mathrm{min}$, and one at the rate of $2330 \mathrm{lb} / \mathrm{min}$. All specimens were cut from plaque $\mathrm{P} 10$ in the $0^{\circ}$ direction. Test results are summarized in Table 7, where the load rates, equivalent strain rates, compressive stiffness, ultimate compressive strength, and failure strain are given together with the corresponding averages and standard deviations.

Table 7. Summary of compressive properties obtained at different constant load rates

\begin{tabular}{lccccc}
\hline $\begin{array}{c}\text { Specimen } \\
\text { No. }\end{array}$ & $\begin{array}{c}\text { Load Rate } \\
\text { (lb/min) }\end{array}$ & $\begin{array}{c}\text { Equivalent Strain } \\
\text { Rate }\left(\mathrm{s}^{-1}\right)\end{array}$ & $\begin{array}{c}\text { Compressive } \\
\text { Stiffness (Msi) }\end{array}$ & $\begin{array}{c}\text { Ultimate } \\
\text { Compressive } \\
\text { Strength (ksi) }\end{array}$ & $\begin{array}{c}\text { Failure } \\
\text { Strain (\%) }\end{array}$ \\
\hline P10-0-3 & 13,980 & $2 \times 10^{-3}$ & 1.30 & -25.3 & -2.79 \\
P10-0-4A & 13,980 & $2 \times 10^{-3}$ & 1.24 & -23.0 & -2.41 \\
P10-0-9 & 2330 & $3 \times 10^{-4}$ & 1.28 & -21.4 & -2.29 \\
P10-0-6 & 7.5 & $10^{-6}$ & 1.27 & -18.5 & -2.09 \\
P10-0-8A & 7.5 & $10^{-6}$ & 1.27 & -13.6 & -1.42 \\
P10-0-6A & 7.5 & $10^{-6}$ & 1.32 & -14.1 & -1.21 \\
& & & & & -19.3 \\
Average & & & 1.28 & -2.04 \\
St. Deviation & & & 0.03 & 4.79 & 0.61 \\
\hline
\end{tabular}

Results are also graphically presented in Fig. 17 where the stress-strain curves obtained at different load rates are shown. The different curves in Fig. 17 are identified by the equivalent strain rates. It is seen in Table 7 and in Fig. 17 that the effect of loading rate on compressive stiffness is negligible. A slight effect of rate on ultimate compressive stress and failure strain may be observed, both quantities decrease somewhat with decreasing load rate. It is important to recognize that a similar rate effect was observed in tension (see Section 3.5). It is noteworthy that compressive stress-strain curves in Fig. 17 compare well with tensile stress-strain curves presented in Fig. 14. 


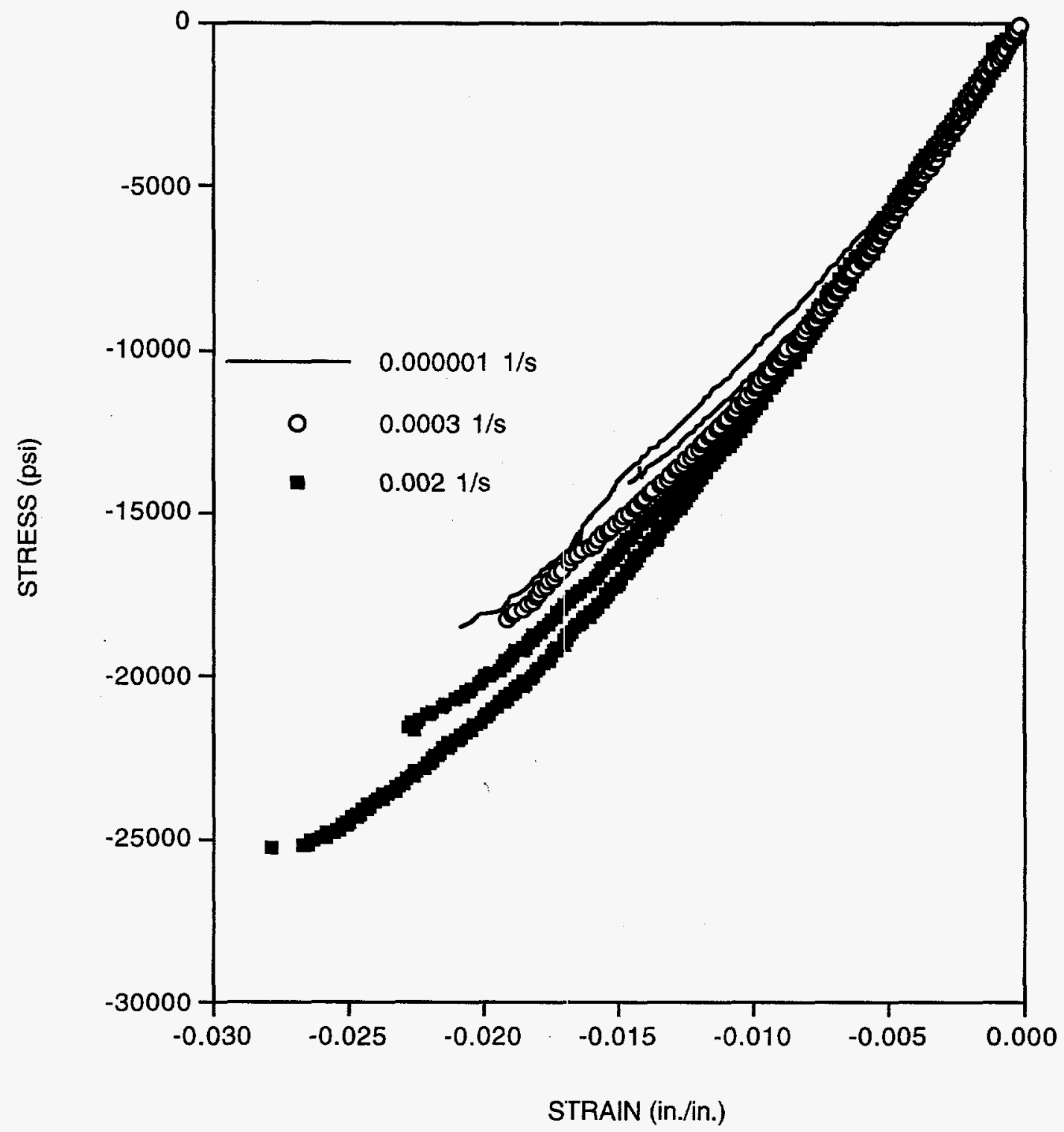

Fig. 17. Compressive stress-strain curves obtained at the load rates of 13980,2330 , and $7.5 \mathrm{lb} / \mathrm{min}$ corresponding to the strain rates of $2 \times 10^{-3}, 3 \times 10^{-4}$, and $10^{-6} \mathrm{~s}^{-1}$, respectively. 


\section{CONCLUDING REMARKS}

\subsection{TENSILE BEHAVIOR}

Assessment of the tensile results suggests the following conclusions.

- The $90^{\circ}$ direction is the "strong" direction, yielding higher average values of stiffiness and UTS. The $90^{\circ}$ average stiffness $\mathrm{E}$ is $23 \%$ higher, and the average UTS is $34 \%$ higher than the corresponding $0^{\circ}$ values.

- Tensile results obtained for the $0^{\circ}$ and $90^{\circ}$ directions form a consistent set of data. The $90^{\circ}$ results generally fall on the high end of the set forming a natural continuation of the $0^{\circ}$ distribution.

- Ultimate tensile strength increases with increasing stiffness, UTS and stiffness can be correlated by a linear law.

- Ultimate tensile strength and stiffness are independent of failure strain.

- Failure strain remains fairly constant for both $0^{\circ}$ and $90^{\circ}$ directions.

Assessment of the proportional limit stress and strain values established for the tensile tests results in the following observations.

- For the reference material, proportional limit stress is equal to $24 \%$ and $22 \%$ of the UTS for the $0^{\circ}$ and $90^{\circ}$ directions, respectively. Proportional limit strain is equal to $18 \%$ of the failure strain for both $0^{\circ}$ and $90^{\circ}$ directions.

- Proportional limit stress increases with increasing UTS and stiffness. While not dramatic, these trends are noticeable and can be represented by linear laws.

- Proportional limit stress is independent of failure strain.

- Proportional limit strain is independent of UTS, stiffness, or failure strain.

- Failure strain and proportional limit strain remain constant for the entire $0^{\circ}-90^{\circ}$ population.

The experimental investigation of damage development in tensile loading as manifested by changes in stiffness leads to the following conclusion.

- No significant changes in stiffness occur below $30 \%$ UTS. Above this threshold stiffness decreases with increasing prior load; this dependence can be described with a linear law.

The investigation of rate dependence of tensile behavior reveals that

- Strain rate sensitivity is apparent, albeit slight at the midrange $\left(10^{-2}\right.$ to $\left.10^{-4} \mathrm{~s}^{-1}\right)$, but it becomes considerable when strain rates approach the dynamic range $\left(10 \mathrm{~s}^{-5}\right)$ and at very slow rates $\left(10^{-6} \mathrm{~s}^{-1}\right)$.

\subsection{COMPRESSIVE BEHAVIOR}

The compressive results presented above demonstrate the following.

- Compressive properties are comparable to tensile properties.

- Conclusions drawn with regards to correlations between tensile strength, stiffness, and failure strain can be extended to compressive properties.

- Proportional limit stresses and strains obtained in compression are comparable to those obtained in tension.

- Observations made regarding proportional limit stress and strain behavioral trends in tension can be extended to compression.

- As in the case of tension, a slight rate sensitivity is observed at the slower quasistatic rates.

- Compressive behavior is comparable to that in tension. Tensile and compressive test results reveal no significant differences between matrix-dominated (observed in compression) and fiber-dominated (observed in tension) behavior at room temperature. 


\section{ACKNOWLEDGEMENT}

The author wishes to acknowledge the contributions of several individuals. D. L. Erdman, L. B. Klett, and R. D. Lomax conducted the EA2 tensile tests. J. M. Corum provided support of this work and many useful discussions. J. M Corum also, along with G. T. Yahr reviewed the final manuscript. 


\section{7}




\section{APPENDIX A. PLAQUE CUTTING RECORDS}

The reported results were obtained on specimens from a total of 30 plaques of the reference composite. These plaques are listed in Table A1, and the specimen cutting plans are shown in Figs. A.1 - A.7.

Table A1. Composite plaque records

\begin{tabular}{ccc}
\hline ORNL No. & ACC No. & Cutting Plan \\
\hline P1 & $227-44-30$ & Fig. A.1 \\
P2 & $227-44-33$ & Fig. A.1 \\
P3 & $227-44-35$ & Fig. A.1 \\
P4 & $227-44-36$ & Fig. A.1 \\
P5 & $227-44-37$ & Fig. A.1 \\
P6 & $227-44-27$ & Fig. A.2 \\
P8 & $227-44-32$ & Fig. A.2 \\
P9 & $227-44-28$ & Fig. A.2 \\
P11 & $227-45-41$ & Fig. A.3 \\
P12 & $227-45-52$ & Fig. A.3 \\
P13 & $227-45-46$ & Fig. A.3 \\
P14 & $227-45-62$ & Fig. A.3 \\
P15 & $227-45-61$ & Fig. A.4 \\
P17 & $227-45-47$ & Fig. A.5 \\
P18 & $227-45-40$ & Fig. A.5 \\
P19 & $16-13-56$ & Fig. A.5 \\
P20 & $16-13-57$ & Fig. A.5 \\
P21 & $16-13-59$ & Fig. A.5 \\
P22 & $16-13-65$ & Fig. A.3 \\
P23 & $16-13-66$ & Fig. A.3 \\
P26 & $16-13-70$ & Fig. A.3 \\
P27 & $16-13-71$ & Fig. A.2 \\
P30 & $16-13-74$ & Fig. A.3 \\
P33 & $16-13-87$ & Fig. A.2 \\
P34 & $16-13-88$ & Fig. A.2 \\
P35 & $16-13-92$ & Fig. A.2 \\
P36 & $16-13-93$ & Fig. A.2 \\
P37 & $16-13-37$ & Fig. A.6 \\
P42 & $16-13-42$ & Fig. A.2 \\
P44 & $16-13-44$ & Fig. A.7 \\
\hline & &
\end{tabular}




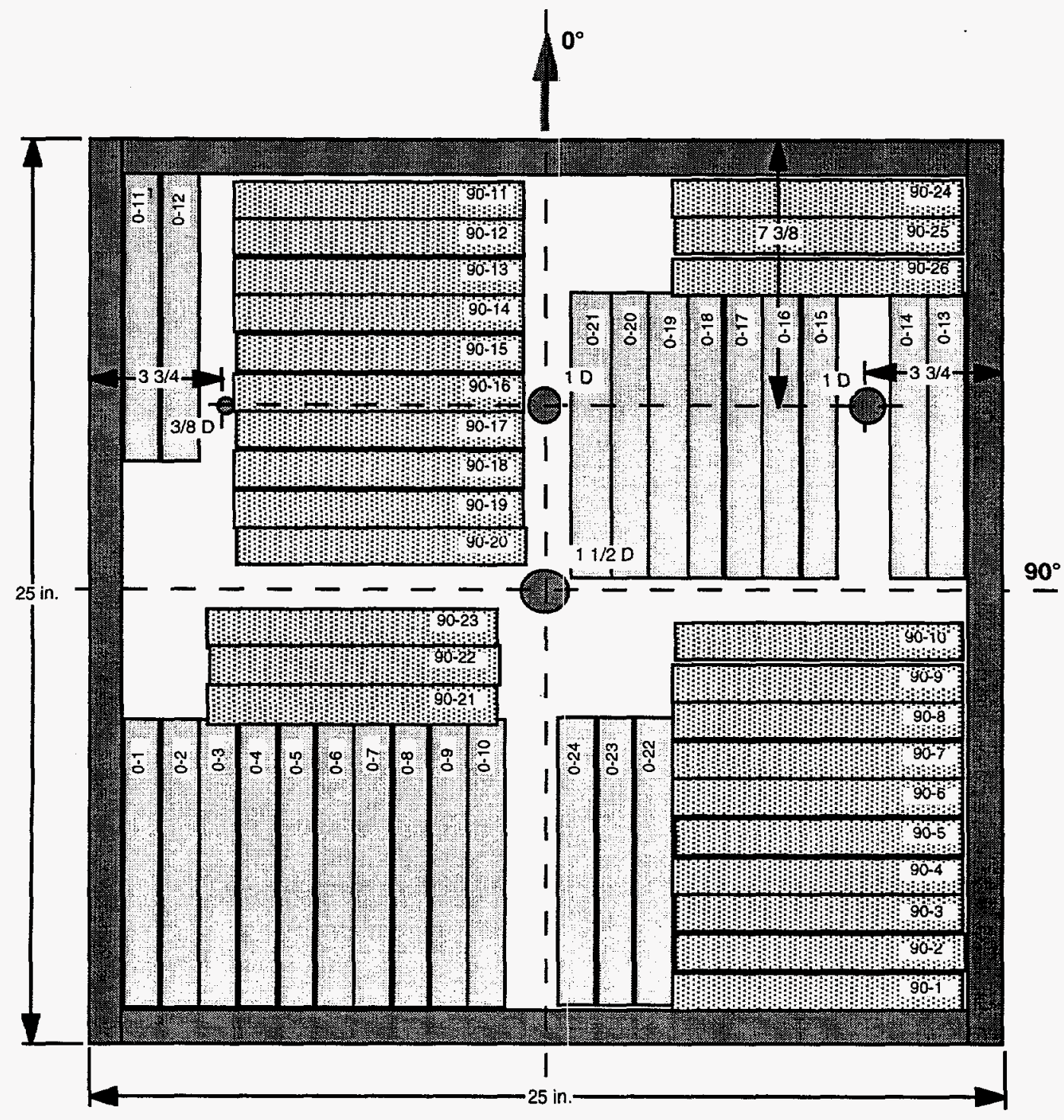

Fig. A.1. Cutting plan for $10^{\circ}$ and $90^{\circ}$ specimens. 


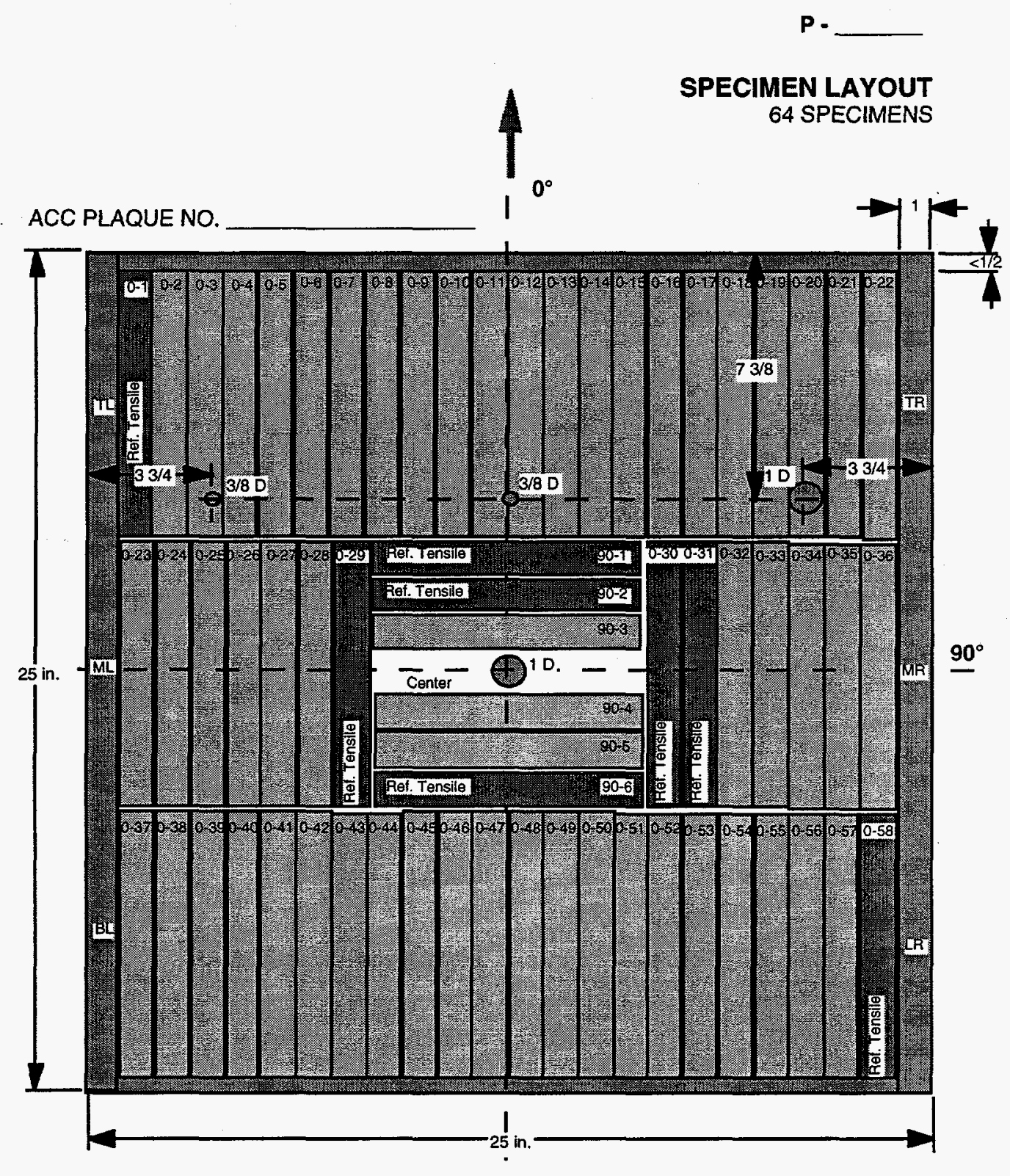

Fig. A.2. Cutting plan for predominantly $0^{\circ}$ test specimens. 


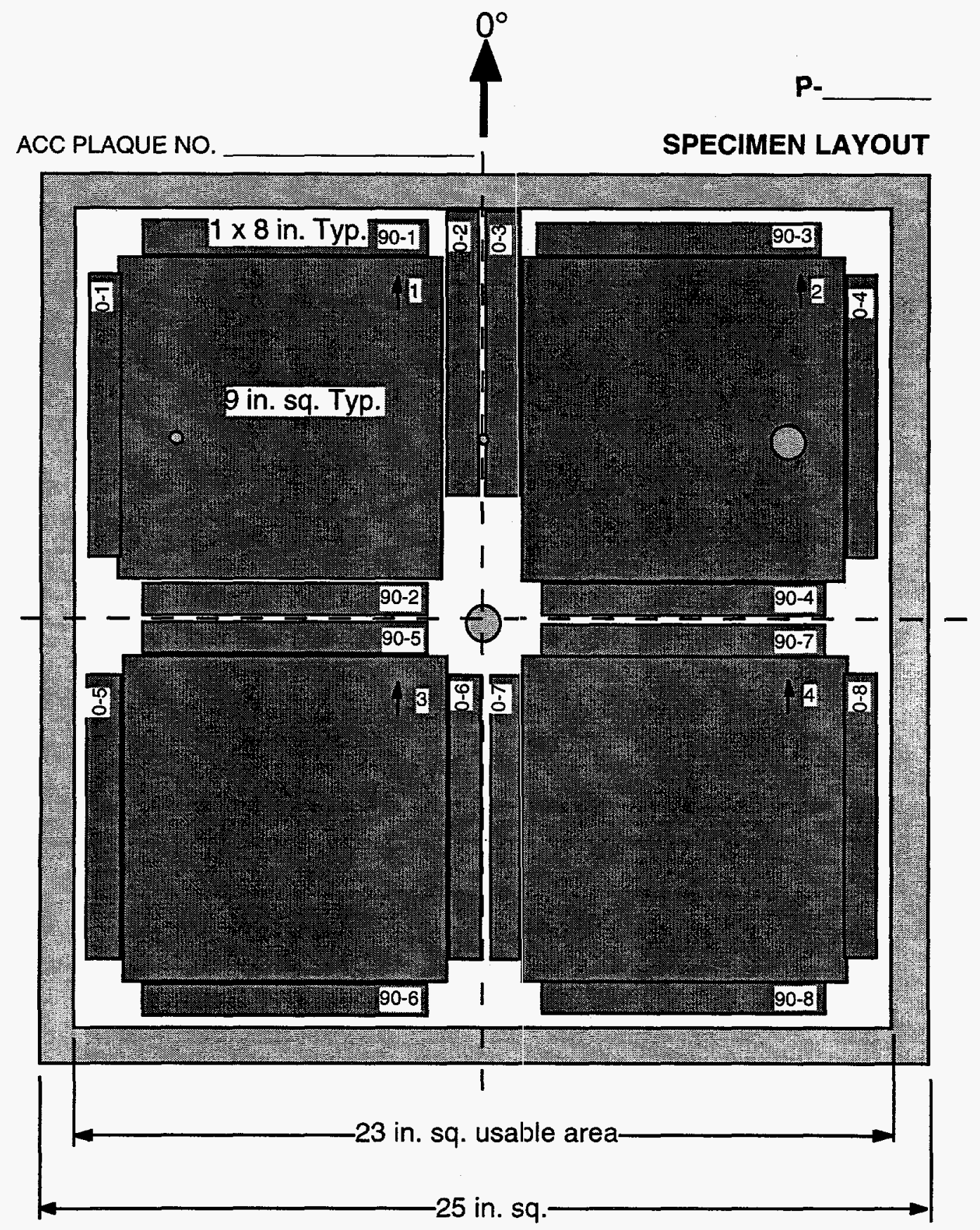

Fig. A.3. Cutting plan for impact test specimens and associated tensile specimens. 


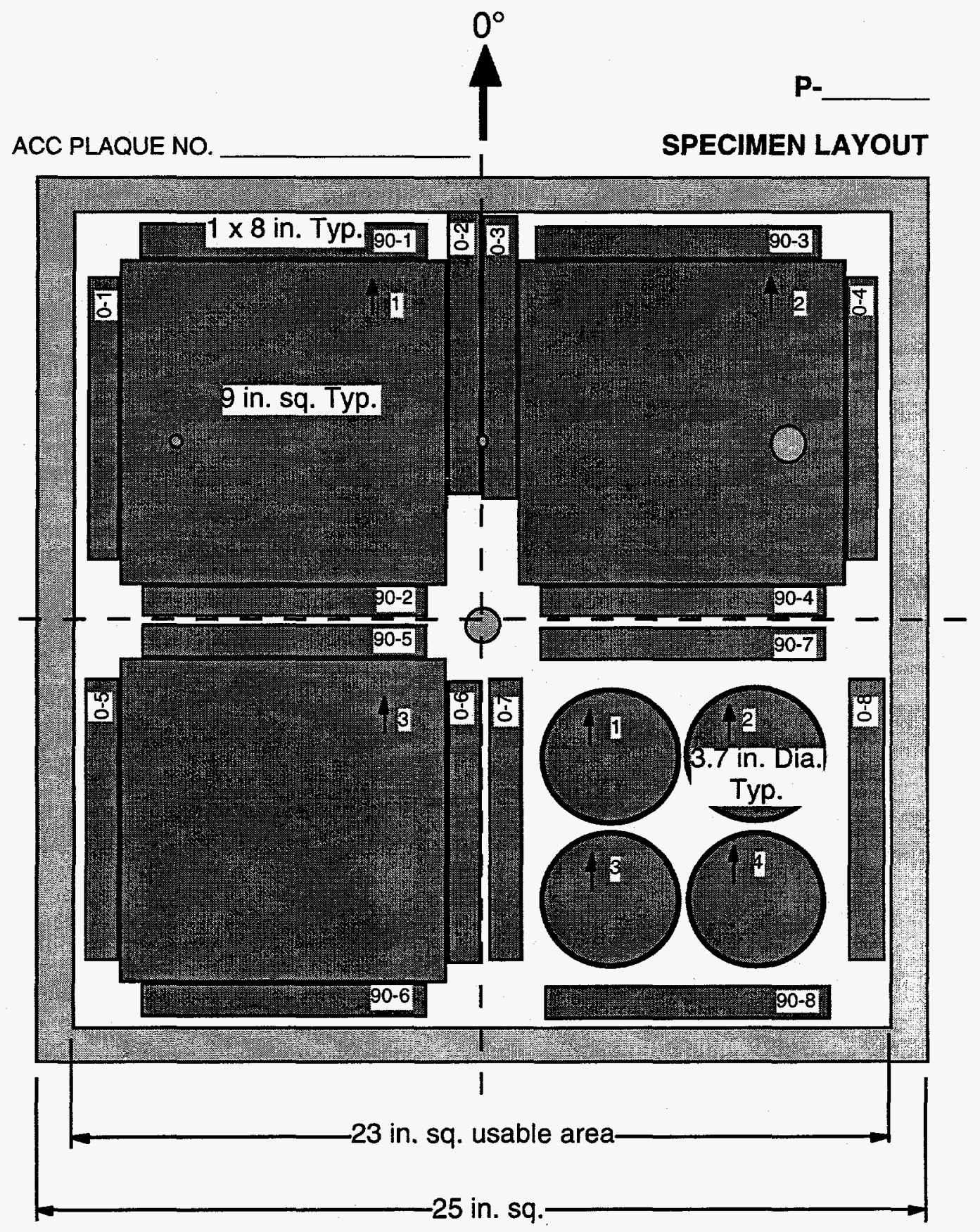

Fig. A.4. Cutting plan for impact and indentation test specimens and associated tensile specimens. 


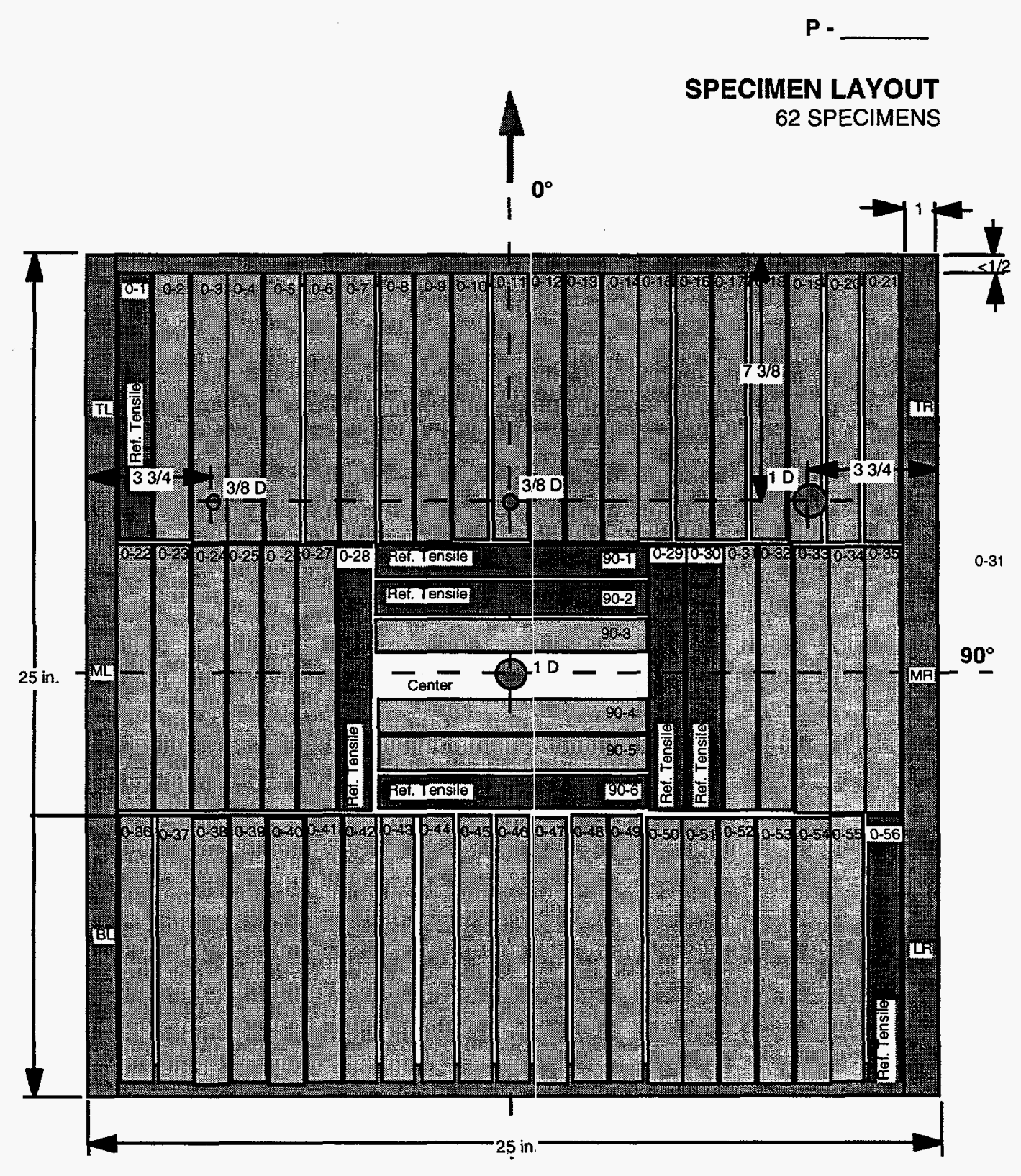

Fig. A.5. Revised cutting plan for predominantly $0^{\circ}$ test specimens. 
P -

SPECIMEN LAYOUT

19 COMPRESSION SPECIMENS

37 SHEAR SPECIMENS

24 BEAM SPECIMENS $(3,5,7,11$ in.)

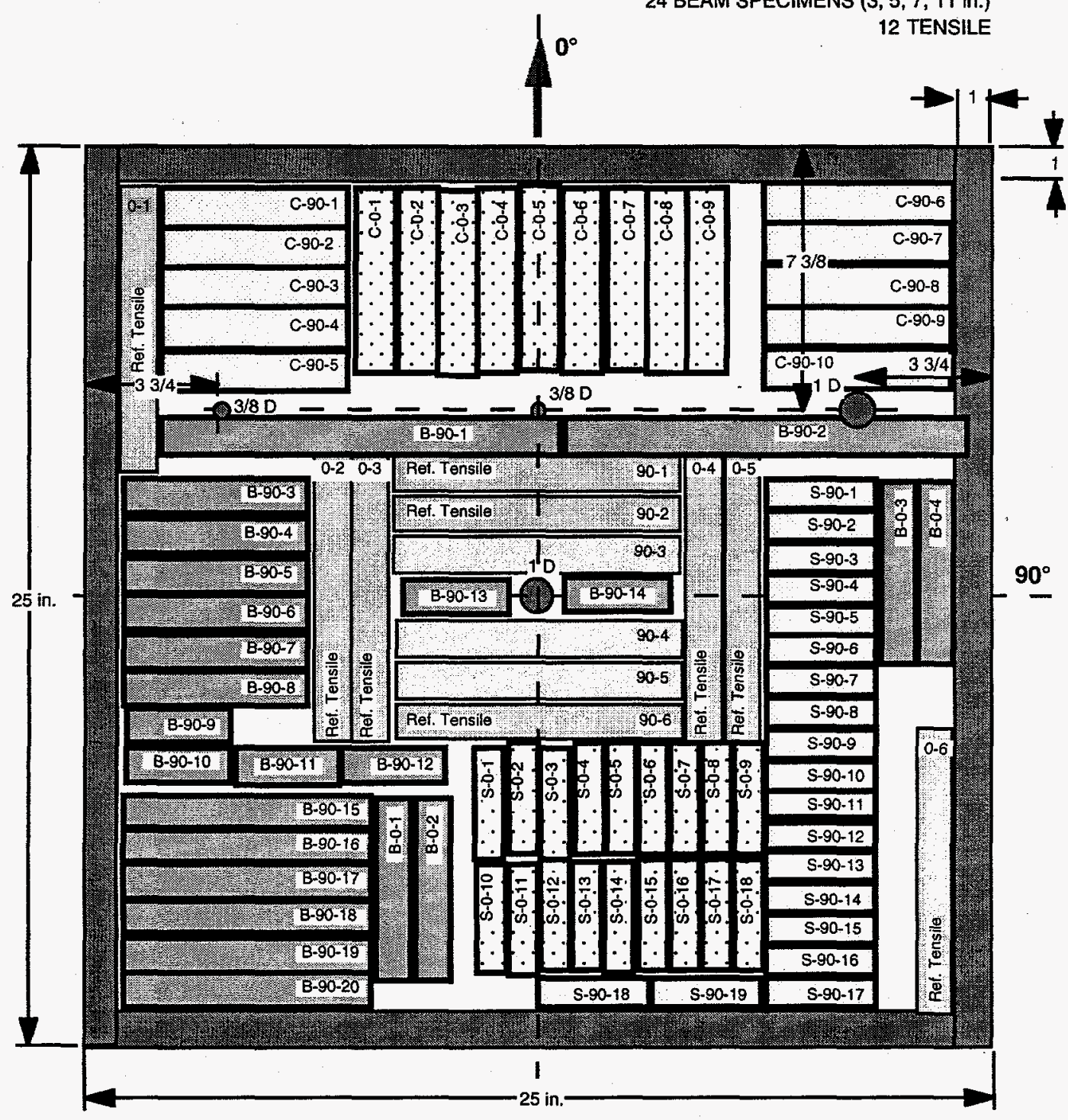

Fig. A.6. Cutting plan for compression, shear, beam, and tensile test specimens. 


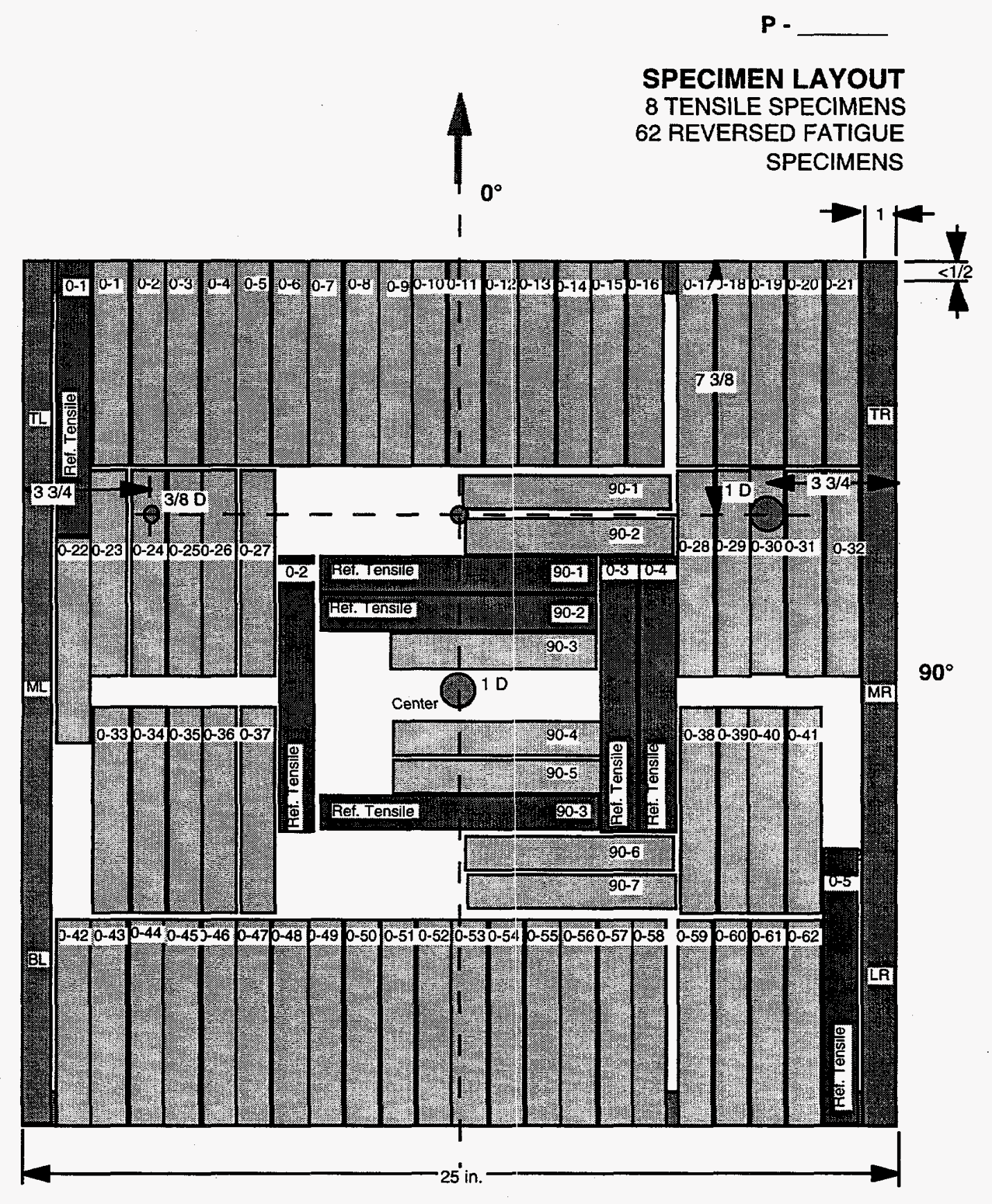

Fig. A.7. Cutting plan for reversed fatigue and associated tensile test specimens. 


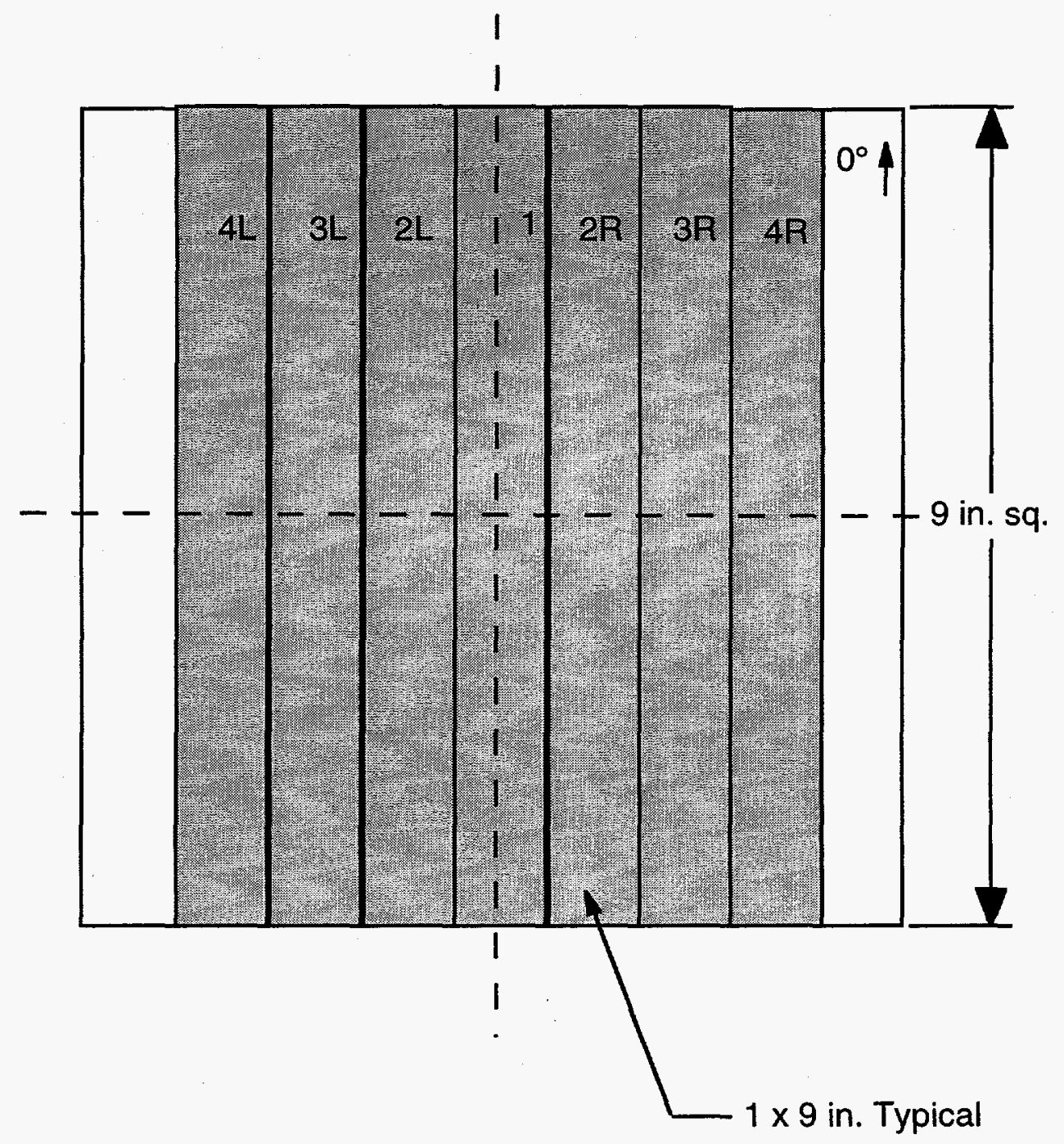

Fig. A.8. Plan for cutting tensile test specimens from impact specimens. 


\section{INTERNAL DISTRIBUTION}

1. R. L. Battiste

2. R. G. Boeman

3. C. R. Brinkman

4. J. M. Corum

5. W. G. Craddick

6. M. Elahi

7. D. L. Erdman

8. W. Ren

\author{
9-13. M. B. Ruggles \\ 10. C. D. Warren \\ 15. Y. J. Weitsman \\ 16. G. T. Yahr \\ 17. R. E. Ziegler \\ 18. ORNL Patent Section \\ 19. ORNL Laboratory Records (RC)
}

\section{EXTERNAL DISTRIBUTION}

20. K. Fielder, The Dow Chemical Company, 2301 N. Brazosport Blvd., B1608, Freeport, Texas 77541-3257.

21-26. E. M. Hagerman, Autmotive Composite Consortium, General Motors, 30500 Mound Road, Box 9055, Warren, Michigan 48090-9055.

27. J. M. Henshaw, Department of Mechanical Engineering, The University of Tulsa, $600 \mathrm{~S}$. College Avenue, Tulsa, Oklahoma 74104-3189.

28. K. Liechti, Engineering Mechanics Research Laboratory, Department of Aerospace Engineering and Engineering Mechanics, The University of Texas at Austin, Austin, Texas 78712.

29. D. N. Robinson, Department of Civil Engineering, The University of Akron, Akron, Ohio 44325-3905.

30. L. V. Smith, Washington State University, School of Mechanics and Materials Engineering, Pullman, Washington 99164-2920.

31. D. C. Worley, Department of Materials Science and Engineering, The University of Tennessee, 427-B Dougherty Engineering Building, Knoxville, Tennessee 37996-2200.

32. J. A. Carpenter, U.S. Department of Energy, 1000 Independence Avenue, SW, Washington, DC 20588.

33. H. E. Clark, U.S. Department of Energy, Oak Ridge Site Office, Oak Ridge, Tennessee 37831 .

34. M. R. Rawlins, U.S. Department of Energy, Oak Ridge Site Office, Oak Ridge, Tennessee 37831 . 